\title{
On the existence of strong solutions to a fluid structure interaction problem with Navier boundary conditions
}

\author{
Imene A. Djebour ${ }^{1}$ and Takéo Takahashi*1 \\ ${ }^{1}$ Université de Lorraine, CNRS, Inria, IECL, F-54000 Nancy
}

March 8, 2019

\begin{abstract}
We consider a fluid-structure interaction system composed by a three-dimensional viscous incompressible fluid and an elastic plate located on the upper part of the fluid boundary. The fluid motion is governed by the Navier-Stokes system whereas we add a damping in the plate equation. We use here Navier-slip boundary conditions instead of the standard no-slip boundary conditions. The main results are the local in time existence and uniqueness of strong solutions of the corresponding system and the global in time existence and uniqueness of strong solutions for small data and if we assume the presence of frictions in the boundary conditions.
\end{abstract}

Keywords: Navier-Stokes system, damped beam equation, strong solutions.

2010 Mathematics Subject Classification. 35Q30, 76D05, 76D03, 74F10

\section{Contents}

\begin{tabular}{lll}
\hline & Introduction & 2 \\
\hline
\end{tabular}

2 Notation $\quad 5$

\begin{tabular}{|lll}
\hline 3 & Change of variables & 7 \\
\hline
\end{tabular}

\begin{tabular}{|lll}
\hline & Regularity properties of the Stokes system & 10
\end{tabular}

$\begin{array}{|ll|}5 & \text { Linear System }\end{array}$

6 Fixed point

6.1 Estimates on the change of variables . . . . . . . . . . . . . . . . . . . . . . . . . . . . 23

6.2 Estimates of $F, G, H \ldots \ldots \ldots \ldots \ldots \ldots$

6.3 Proof of Theorem $6.1 \ldots \ldots \ldots \ldots \ldots \ldots \ldots$

*Takéo Takahashi acknowledges the support of the Agence Nationale de la Recherche, project IFSMACS, ANR-15-CE40-0010 


\section{Introduction}

The aim of this work is to analyze the interaction between a viscous incompressible fluid and a viscous elastic plate. Let us start by presenting the corresponding model. We denote by $\omega$ the rectangular torus

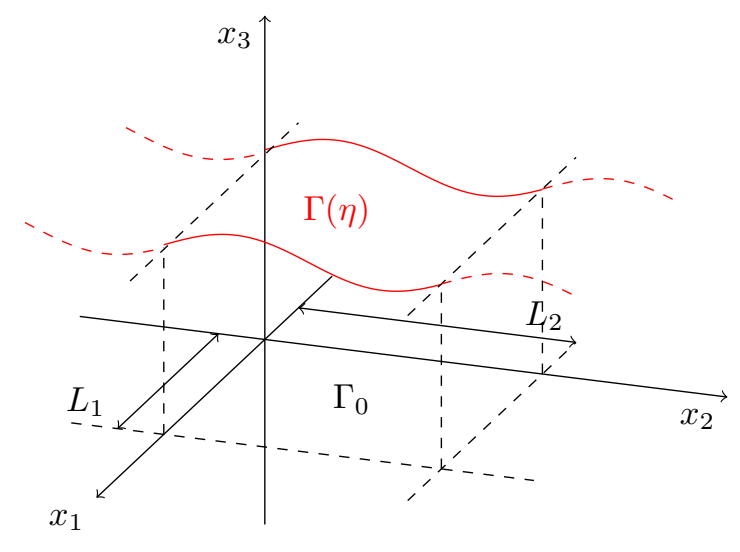

Figure 1: Configuration of the domain at time $t$.

$$
\omega=\left(\mathbb{R} / L_{1} \mathbb{Z}\right) \times\left(\mathbb{R} / L_{2} \mathbb{Z}\right) \quad L_{1}>0, L_{2}>0 .
$$

For any function $\eta: \omega \rightarrow(-1, \infty)$, we define (see Figure 1)

$$
\begin{aligned}
\Omega(\eta) & =\left\{\left(x_{1}, x_{2}, x_{3}\right) \in \omega \times \mathbb{R} \mid 0<x_{3}<1+\eta\left(x_{1}, x_{2}\right)\right\}, \\
\Gamma(\eta) & =\left\{\left(x_{1}, x_{2}, x_{3}\right) \in \omega \times \mathbb{R} \mid x_{3}=1+\eta\left(x_{1}, x_{2}\right)\right\}, \\
\Gamma_{0} & =\omega \times\{0\} .
\end{aligned}
$$

In particular

$$
\partial \Omega(\eta)=\Gamma(\eta) \cup \Gamma_{0} .
$$

We consider the following system describing the evolution of the fluid governed by the incompressible NavierStokes equations, and the movement of the elastic plate

$$
\left\{\begin{array}{rl}
\partial_{t} U+(U \cdot \nabla) U-\nabla \cdot \mathbb{T}(U, P)=0 & t>0, x \in \Omega(\eta(t, \cdot)), \\
\nabla \cdot U=0 & t>0, x \in \Omega(\eta(t, \cdot)), \\
\partial_{t t} \eta+\alpha \Delta^{2} \eta-\kappa \Delta \eta+\sigma \eta-\delta \Delta \partial_{t} \eta=\widetilde{\mathbb{H}}_{\eta}(U, P) \quad t>0, s \in \omega .
\end{array}\right.
$$

In the above system, we have denoted by $U$ the fluid velocity, $P$ the fluid pressure and $\eta$ the transversal plate displacement.

The Cauchy stress tensor $\mathbb{T}(U, P)$ is defined by

$$
\mathbb{T}(U, P)=-P I_{3}+2 \nu D(U), \quad D(U)_{i, j}=\frac{1}{2}\left(\frac{\partial U_{i}}{\partial x_{j}}+\frac{\partial U_{j}}{\partial x_{i}}\right) .
$$

The function $\widetilde{\mathbb{H}}_{\eta}$ is the fluid strain on the structure and is defined by

$$
\widetilde{\mathbb{H}}_{\eta}(U, P)=-\sqrt{1+|\nabla \eta|^{2}}\left(\mathbb{T}(U, P) n \cdot e_{3}\right) .
$$

We assume

$$
\nu>0, \quad \alpha>0, \quad \sigma \geqslant 0, \quad \kappa \geqslant 0 \text { and } \delta \geqslant 0 .
$$


These constants correspond respectively to the rigidity $(\alpha)$, the stretching $(\kappa)$, the damping on the structure $(\delta)$ and the viscosity $(\nu)$.

We have denoted by $n$ the unitary exterior normal of $\partial \Omega(\eta)$ :

$$
n=-e_{3} \text { on } \Gamma_{0},
$$

and on $\Gamma(\eta)$ :

$$
n(s, 1+\eta(s))=\frac{N(s, 1+\eta(s))}{|N(s, 1+\eta(s))|}, \quad \text { where } \quad N(s, 1+\eta(s))=\left(\begin{array}{c}
-\partial_{s_{1}} \eta(s) \\
-\partial_{s_{2}} \eta(s) \\
1
\end{array}\right), \quad s \in \omega
$$

Here and in what follows, $|\cdot|$ denotes the Euclidian norm of $\mathbb{R}^{k}, k \geqslant 1$.

We complete (1.3) by the Navier slip boundary conditions. In order to write these boundary conditions, we need to introduce some notations. We denote by $a_{n}$ and $a_{\tau}$ the normal and the tangential parts of $a \in \mathbb{R}^{3}$ :

$$
a_{n}=(a \cdot n) n, \quad a_{\tau}=a-a_{n}=-n \times(n \times a) .
$$

Then, our boundary conditions write as follows

$$
\left\{\begin{aligned}
U_{n} & =0 \quad t>0, x \in \Gamma_{0}, \\
{[2 D(U) n]_{\tau}+\beta_{1} U_{\tau} } & =0 \quad t>0, x \in \Gamma_{0}, \\
\left(U(t, s, 1+\eta(t, s))-\partial_{t} \eta(t, s) e_{3}\right)_{n} & =0 \quad t>0, s \in \omega, \\
{[2 D(U) n]_{\tau}(t, s, 1+\eta(t, s))+\beta_{2}\left(U\left(t, s, 1+\eta(t, s)-\partial_{t} \eta(t, s) e_{3}\right)_{\tau}\right.} & =0 \quad t>0, s \in \omega .
\end{aligned}\right.
$$

In what follows, we write the above equations in the following more compact way

$$
\left\{\begin{array}{rl}
U_{n}=0 & t>0, x \in \Gamma_{0} \\
{\left[2 \nu D(U) n+\beta_{1} U\right]_{\tau}=0} & t>0, x \in \Gamma_{0} \\
\left(U-\partial_{t} \eta e_{3}\right)_{n}=0 & t>0, x \in \Gamma(\eta) \\
{\left[2 \nu D(U) n+\beta_{2}\left(U-\partial_{t} \eta e_{3}\right)\right]_{\tau}=0} & t>0, x \in \Gamma(\eta) .
\end{array}\right.
$$

We assume that the friction coefficients $\beta_{1}$ and $\beta_{2}$ are constants satisfying

$$
\beta_{1} \geqslant 0, \quad \beta_{2} \geqslant 0
$$

These boundary conditions can be compared with the standard no-slip boundary conditions usually considered with the Navier-Stokes system. In our case, these conditions would write as

$$
\left\{\begin{array}{rl}
U=0 & t>0, x \in \Gamma_{0} \\
U=\partial_{t} \eta e_{3} & t>0, x \in \Gamma(\eta) .
\end{array}\right.
$$

The Navier slip boundary condition was proposed by Navier in 1823 [28] and is relevant in several physical contexts, see for instance [24, 35, 22,.

To complete the system $[1.3,(1.8)$, we add the following initial conditions

$$
\left\{\begin{aligned}
\eta(0, \cdot)=\eta^{0} & \text { in } \omega \\
\partial_{t} \eta(0, \cdot)=\eta^{1} & \text { in } \omega \\
U(0, \cdot)=U^{0} & \text { in } \Omega\left(\eta^{0}\right)
\end{aligned}\right.
$$

Let us remark that we don't need to consider boundary conditions on the "lateral" boundaries since we work with the torus $\omega$ (see (1.1) and (1.2). This means that we are considering periodic boundary conditions for $U$, $P$ and $\eta$ :

$$
\begin{array}{cl}
U\left(t, x_{1}+L_{1}, x_{2}, x_{3}\right)=U\left(t, x_{1}, x_{2}, x_{3}\right), & U\left(t, x_{1}, x_{2}+L_{2}, x_{3}\right)=U\left(t, x_{1}, x_{2}, x_{3}\right), \\
\eta\left(t, s_{1}+L_{1}, s_{2}\right)=\eta\left(t, s_{1}, s_{2}\right), & \eta\left(t, s_{1}, s_{2}+L_{2}\right)=\eta\left(t, s_{1}, s_{2}\right),
\end{array}
$$


and a similar relations for $P$.

Several works have been devoted to the study of the system $(1.3),(1.10)$ with the Dirichlet boundary conditions (1.9): existence of strong solutions ([3], [23]), feedback stabilization ([30], [2]), global existence of strong solutions $([15])$. Let us point out that in this latter work, the authors manage to obtain in particular that there is no contact between the plate and the bottom of the domain in finite time for the system $\sqrt{1.3 p}, \sqrt{1.9 p},(1.10)$. This result, as previous works on fluid-structure interaction systems, shows that the standard no-slip boundary conditions may lead to some paradoxal results as the distance between two structures is going to 0: in the case of rigid bodies immersed into a viscous incompressible fluid, it is shown that in particular geometries there is no contact in finite time of two structures ([18], [19]) and in general, if there is contact, then it occurs with null relative velocity and null relative acceleration (31]). In [9] and [10], the author considered boundary conditions involving the pressure. Here, our aim is to analyze the same system (1.3) with the Navier-slip boundary conditions (1.8) instead of the Dirichlet boundary conditions. Such a system was already considered in [17] and [27] where the existence of weak solutions is proved in dimension 2 (global existence as long as the deformable structure does not touch the fixed bottom). The uniqueness of weak solutions for this system has been obtained in [16].

Our objective is to prove the existence and uniqueness of strong solutions for small time or for small data. This is the first work on strong solutions for such a system in the case of Navier-slip boundary conditions and to our knowledge, it is also the first work on strong solutions for this kind of systems in the 3D case.

In the case where the structures are rigid bodies immersed into a viscous incompressible fluid, several authors have already considered the Navier-slip boundary conditions: existence of weak solutions [29] and [12, existence of contact in finite time [13], existence of strong solutions and study of contacts in finite time [36], uniqueness of weak solutions [7]. Let us also mention the work of [8] where they consider a nonlinear boundary condition of Tresca's type.

The main result of this article is

\section{Theorem 1.1.}

1. Assume $\beta_{i} \geqslant 0$ for $i=1,2$ and (1.4). Suppose $\eta^{0} \in H^{3}(\omega), \eta^{1} \in H^{1}(\omega)$ and $U^{0} \in\left[H^{1}\left(\Omega\left(\eta^{0}\right)\right)\right]^{3}$ such that

$$
1+\eta^{0}>0, \quad \nabla \cdot U^{0}=0 \quad \text { in } \Omega\left(\eta^{0}\right), \quad\left(U^{0}-\eta^{1} e_{3}\right)_{n}=0 \quad \text { on } \Gamma\left(\eta^{0}\right), \quad U_{n}^{0}=0 \quad \text { on } \Gamma_{0} .
$$

There exists a time $T_{0}$ such that the system (1.3), 1.8, 1.10) admits a unique strong solution $(U, P, \eta)$ on $\left(0, T_{0}\right)$ :

$$
\begin{gathered}
\eta \in L^{2}\left(0, T_{0} ; H^{4}(\omega)\right) \cap C^{0}\left(\left[0, T_{0}\right] ; H^{3}(\omega)\right) \cap H^{1}\left(0, T_{0} ; H^{2}(\omega)\right) \cap C^{1}\left(\left[0, T_{0}\right] ; H^{1}(\omega)\right) \cap H^{2}\left(0, T_{0} ; L^{2}(\omega)\right), \\
U \in L^{2}\left(0, T_{0} ;\left[H^{2}(\Omega(\eta(t))]^{3}\right) \cap C^{0}\left(\left[0, T_{0}\right] ;\left[H^{1}(\Omega(\eta(t)))\right]^{3}\right) \cap H^{1}\left(0, T_{0} ;\left[L^{2}(\Omega(\eta(t)))\right]^{3}\right),\right. \\
\nabla P \in L^{2}\left(0, T_{0} ;\left[L^{2}(\Omega(\eta(t)))\right]^{3}\right) .
\end{gathered}
$$

2. Assume $\beta_{i} \geqslant 0$ for $i=1,2$ with $\beta_{1}+\beta_{2}>0$ and (1.4). There exist $\gamma_{0}>0$ and $R_{0}>0$ such that if $\eta^{0} \in H^{3}(\omega), \eta^{1} \in H^{1}(\omega)$ and $U^{0} \in\left[H^{1}\left(\Omega\left(\eta^{0}\right)\right)\right]^{3}$ satisfy

$$
1+\eta^{0}>0, \quad \nabla \cdot U^{0}=0 \quad \text { in } \Omega\left(\eta^{0}\right), \quad\left(U^{0}-\eta^{1} e_{3}\right)_{n}=0 \quad \text { on } \Gamma\left(\eta^{0}\right), \quad U_{n}^{0}=0 \quad \text { on } \Gamma_{0} .
$$

and

$$
\left\|U^{0}\right\|_{\left[H^{1}(\Omega)\right]^{3}}+\left\|\eta^{0}\right\|_{H^{3}(\omega)}+\left\|\eta^{1}\right\|_{H^{1}(\omega)} \leqslant R_{0},
$$

then the system (1.3), (1.8), (1.10) admits a unique strong solution $(U, P, \eta)$ on $(0, \infty)$ :

$$
\begin{gathered}
\eta \in L_{\gamma}^{2}\left(0, \infty ; H^{4}(\omega)\right) \cap B C_{\gamma}^{0}\left([0, \infty] ; H^{3}(\omega)\right) \cap H_{\gamma}^{1}\left(0, \infty ; H^{2}(\omega)\right) \cap B C_{\gamma}^{1}\left([0, \infty] ; H^{1}(\omega)\right) \cap H_{\gamma}^{2}\left(0, \infty ; L^{2}(\omega)\right), \\
U \in L_{\gamma}^{2}\left(0, \infty ;\left[H^{2}(\Omega(\eta(t))]^{3}\right) \cap B C_{\gamma}^{0}\left([0, \infty] ;\left[H^{1}(\Omega(\eta(t)))\right]^{3}\right) \cap H_{\gamma}^{1}\left(0, \infty ;\left[L^{2}(\Omega(\eta(t)))\right]^{3}\right),\right. \\
\nabla P \in L_{\gamma}^{2}\left(0, \infty ;\left[L^{2}(\Omega(\eta(t)))\right]^{3}\right),
\end{gathered}
$$

for $\gamma \in\left[0, \gamma_{0}\right]$. 
In the above statement, the spaces $L^{p}, H^{s}$ are the classical Lebesgue, Sobolev spaces. We use the notation $B C^{0}=C^{0} \cap L^{\infty}$ and $B C^{1}=C^{1} \cap W^{1, \infty}$. The notation $\cdot_{\gamma}$ is explained below in $(2.2$, 2.3) and corresponds to an exponential decay of order $\gamma$. Finally, the notation $L^{2}\left(0, T ; H^{1}(\Omega(\eta(t)))\right)$ corresponds to the fact that the fluid velocity and pressure are written in a moving domain depending on $\eta$. To obtain our result, we thus need to use a change of variables for $U$ and $P$ and the fluid velocity and pressure after change of variables are obtained in spaces of the form $L^{2}\left(0, T ; H^{1}(\Omega)\right)$ with a fixed $\Omega$. The precise definition of strong solutions is given in Section 3 (Definition 3.1) and we reformulate the above result in a more precise way in Theorem 6.1

Remark 1.2. We can write a bi-dimensional version of the system 1.3), 1.8, , 1.10 and for such a system, one can prove a similar result as Theorem 1.1. In fact, in that case, one could obtain a global in time existence of strong solutions up to a possible contact between the beam and the bottom of the domain by following the arguments in [15].

Remark 1.3. For the sake of simplicity in the proof of Theorem 1.1 and in the remaining part of this article, we assume $\kappa=\sigma=0$ since these constants do not play any role in the analysis.

The plan of this paper is as follows: In Section 2, we give some notation. In Section 3, we remap the problem into a fixed domain using a change of variables like it was introduced in [21, and we restate Theorem 1.1. We obtain some regularity properties of the Stokes system in domains of class $H^{3}$ in Section 4 . In Section 5 , we study the linearized problem by writing it as an evolution equation. We prove in particular that the associated semigroup is analytic and in Section 6, we prove the main result using a fixed-point argument.

\section{Notation}

During the course of our analysis, we will use some functional spaces that we introduce in this section.

First, let us note that due to the incompressibility of the fluid and to the boundary conditions $[1.8]_{1}$ and $1.8]_{3}$, we have

$$
\frac{d}{d t} \int_{\omega} \eta d s=0
$$

For simplicity, we assume throughout the paper that

$$
\int_{\omega} \eta^{0} d s=0
$$

so that

$$
\int_{\omega} \eta(t, \cdot) d s=0 \quad(t \geqslant 0)
$$

It yields to consider the following space

$$
L_{0}^{2}(\omega)=\left\{\xi \in L^{2}(\omega) \mid \int_{\omega} \xi d s=0\right\},
$$

and the orthogonal projection $M: L^{2}(\omega) \rightarrow L_{0}^{2}(\omega)$. Applying $M$ on the plate equation $(1.3) 3$, we find

$$
\partial_{t t} \eta+A_{1} \eta+A_{2} \partial_{t} \eta=\mathbb{H}_{\eta}(U, P),
$$

where

$$
\begin{array}{ll}
A_{1} \eta=\alpha \Delta^{2} \eta, & \mathcal{D}\left(A_{1}\right)=H^{4}(\omega) \cap L_{0}^{2}(\omega), \\
A_{2} \eta=-\delta \Delta \eta, & \mathcal{D}\left(A_{2}\right)=H^{2}(\omega) \cap L_{0}^{2}(\omega)
\end{array}
$$

and

$$
\mathbb{H}_{\eta}(U, P)=M\left(\widetilde{\mathbb{H}}_{\eta}(U, P)\right)
$$


The projection of 1.3$]_{3}$ onto $L_{0}^{2}(\omega)^{\perp}$ leads to impose the choice of the constant normalizing the pressure, see for instance [15].

We denote by $H^{s}(0, T ; \mathfrak{X})$ the usual Sobolev spaces with values in a Banach space $\mathfrak{X}$. For $s>0, s \notin \mathbb{N}$, the norm of these spaces can be defined by using

$$
\lfloor\xi\rfloor_{s, 2,(0, T), \mathfrak{X}}=\left(\int_{(0, T) \times(0, T)} \frac{\left\|\xi(t)-\xi\left(t^{\prime}\right)\right\|_{\mathfrak{X}}^{2}}{\left|t-t^{\prime}\right|^{2 s+1}} d t d t^{\prime}\right)^{1 / 2} .
$$

More precisely, the norm $\|\cdot\|_{H^{s}(0, T ; \mathfrak{X})}$ for $s \in(0,1)$ is given by

$$
\|\xi\|_{H^{s}(0, T ; \mathfrak{X})}=\left(\|\xi\|_{L^{2}(0, T ; \mathfrak{x})}^{2}+\lfloor\xi\rfloor_{s, 2,(0, T), \mathfrak{X}}^{2}\right)^{1 / 2} .
$$

We recall (see [6]) that if $s \in\left(\frac{1}{2}, 1\right)$, then the norm $\lfloor\cdot\rfloor_{s, 2,(0, T), \mathfrak{x}}$ is equivalent to the norm defined in 2.1$]$ in the space $\left\{\xi \in H^{s}(0, T ; \mathfrak{X}) \mid \xi(0)=0\right\}$.

Let $\mathfrak{X}_{1}, \mathfrak{X}_{2}$ be two Banach spaces endowed with the norm $\|\cdot\|_{\mathfrak{X}_{1}}$ respectively $\|\cdot\|_{\mathfrak{X}_{2}}$. For $s \geqslant 0$, we define the following space

$$
W^{s}\left(0, T ; \mathfrak{X}_{1}, \mathfrak{X}_{2}\right)=\left\{v \in L^{2}\left(0, T ; \mathfrak{X}_{1}\right) \mid v \in H^{s}\left(0, T ; \mathfrak{X}_{2}\right)\right\},
$$

endowed with norm

$$
\|\cdot\|_{W^{s}\left(0, T ; \mathfrak{X}_{1}, \mathfrak{X}_{2}\right)}=\|\cdot\|_{L^{2}\left(0, T ; \mathfrak{X}_{1}\right)}+\|\cdot\|_{H^{s}\left(0, T ; \mathfrak{X}_{2}\right)} \cdot
$$

For $s=1$, we will denote $W^{1}\left(0, T ; \mathfrak{X}_{1}, \mathfrak{X}_{2}\right)$ by $W\left(0, T ; \mathfrak{X}_{1}, \mathfrak{X}_{2}\right)$.

For $\gamma>0$, we also consider the spaces

$$
L_{\gamma}^{p}\left(0, \infty ; \mathfrak{X}_{1}\right)=\left\{v \in L^{p}\left(0, \infty ; \mathfrak{X}_{1}\right) ; t \mapsto v_{\gamma}(t)=e^{\gamma t} v(t) \in L^{p}\left(0, \infty ; \mathfrak{X}_{1}\right)\right\}, \quad p \in[1,+\infty],
$$

and

$$
W_{\gamma}^{s}\left(0, \infty ; \mathfrak{X}_{1}, \mathfrak{X}_{2}\right)=\left\{v \in W^{s}\left(0, \infty ; \mathfrak{X}_{1}, \mathfrak{X}_{2}\right) ; t \mapsto v_{\gamma}(t)=e^{\gamma t} v(t) \in W^{s}\left(0, \infty ; \mathfrak{X}_{1}, \mathfrak{X}_{2}\right)\right\}
$$

For these spaces, we use the norms defined by

$$
\begin{aligned}
\|v\|_{L_{\gamma}^{p}\left(0, \infty ; \mathfrak{x}_{1}\right)} & =\left\|v_{\gamma}\right\|_{L^{p}\left(0, \infty ; \mathfrak{X}_{1}\right)}, \\
\|v\|_{W_{\gamma}^{s}\left(0, \infty ; \mathfrak{X}_{1}, \mathfrak{X}_{2}\right)} & =\left\|v_{\gamma}\right\|_{W^{s}\left(0, \infty ; \mathfrak{X}_{1}, \mathfrak{X}_{2}\right)} .
\end{aligned}
$$

In what follows, we set

$$
\Omega=\Omega\left(\eta^{0}\right)
$$

for the local existence and

$$
\Omega=\Omega(0)
$$

for the global existence.

In order to differentiate the normal or the normal and tangential component of a vector $v$ in $\Omega$ and in $\Omega(t)$, we use the notation $n_{0}, v_{n_{0}}$ and $v_{\tau_{0}}$ for the configuration $\Omega$.

We denote by

$$
\mathcal{D}_{\sigma}(\Omega)=\left\{\phi \in\left[C_{0}^{\infty}(\Omega)\right]^{3}, \operatorname{div} \phi=0\right\} .
$$

the space of infinitely differentiable functions with free divergence in $\Omega$ with compact support .

Let us also define the following space

$$
\mathcal{X}_{T}=W\left(0, T ;\left[H^{2}(\Omega)\right]^{3},\left[L^{2}(\Omega)\right]^{3}\right) \times L^{2}\left(0, T ; H^{1}(\Omega) / \mathbb{R}\right) \times W^{2}\left(0, T ; \mathcal{D}\left(A_{1}\right), L_{0}^{2}(\omega)\right),
$$


endowed with the norm

$$
\begin{gathered}
\|(u, p, \eta)\|_{\mathcal{X}_{T}}=\|u\|_{W\left(0, T ;\left[H^{2}(\Omega)\right]^{3},\left[L^{2}(\Omega)\right]^{3}\right)}+\|u\|_{L^{\infty}\left(0, T ;\left[H^{1}(\Omega)\right]^{3}\right)}+\|\nabla p\|_{L^{2}\left(0, T,\left[L^{2}(\Omega)\right]^{3}\right)} \\
+\|\eta\|_{W^{2}\left(0, T ; \mathcal{D}\left(A_{1}\right), L_{0}^{2}(\omega)\right)}+\|\eta\|_{L^{\infty}\left(0, T ; H^{3}(\omega)\right)}+\left\|\partial_{t} \eta\right\|_{L^{\infty}\left(0, T ; H^{1}(\omega)\right)} .
\end{gathered}
$$

If $T=+\infty$ and $\gamma \geqslant 0$, we will write

$$
\mathcal{X}_{\infty, \gamma}=W_{\gamma}\left(0, \infty ;\left[H^{2}(\Omega)\right]^{3},\left[L^{2}(\Omega)\right]^{3}\right) \times L_{\gamma}^{2}\left(0, \infty ; H^{1}(\Omega) / \mathbb{R}\right) \times W_{\gamma}^{2}\left(0, \infty ; \mathcal{D}\left(A_{1}\right), L_{0}^{2}(\omega)\right),
$$

endowed with the norm

$$
\begin{aligned}
\|(u, p, \eta)\|_{\mathcal{X}_{\infty, \gamma}}=\|u\|_{W_{\gamma}\left(0, \infty ;\left[H^{2}(\Omega)\right]^{3},\left[L^{2}(\Omega)\right]^{3}\right)}+\|u\|_{L_{\gamma}^{\infty}\left(0, \infty ;\left[H^{1}(\Omega)\right]^{3}\right)}+\|\nabla p\|_{L_{\gamma}^{2}\left(0, \infty,\left[L^{2}(\Omega)\right]^{3}\right)} \\
+\|\eta\|_{W_{\gamma}^{2}\left(0, \infty ; \mathcal{D}\left(A_{1}\right), L_{0}^{2}(\omega)\right)}+\|\eta\|_{L_{\gamma}^{\infty}\left(0, \infty ; H^{3}(\omega)\right)}+\left\|\partial_{t} \eta\right\|_{L_{\gamma}^{\infty}\left(0, \infty ; H^{1}(\omega)\right)} .
\end{aligned}
$$

To write the boundary conditions, we also introduce the operator $\mathcal{T}$ defined as follows (see [2]):

$$
\mathcal{T}_{\eta^{0}} \xi(y)=\left\{\begin{array}{ccc}
0 & \text { if } & y \in \Gamma_{0}, \\
\xi(s) e_{3} & \text { if } & y=\left(s, 1+\eta^{0}(s)\right) \in \Gamma\left(\eta^{0}\right) .
\end{array}\right.
$$

We can verify that $\mathcal{T}_{\eta^{0}} \in \mathcal{L}\left(L^{2}(\omega) ;\left[L^{2}(\partial \Omega)\right]^{3}\right)$ and that

$$
\mathcal{T}_{\eta^{0}}^{*} \zeta=\sqrt{1+\left|\nabla \eta^{0}\right|^{2}} \zeta \cdot e_{3}, \quad \forall \zeta \in\left[L^{2}(\partial \Omega)\right]^{3} .
$$

We set

$$
\mathcal{T}=\mathcal{T}_{\eta^{0}} M
$$

We also define

$$
\beta=\left\{\begin{array}{llc}
\beta_{1} & \text { if } \quad y \in \Gamma_{0} \\
\beta_{2} & \text { if } \quad y \in \Gamma\left(\eta^{0}\right) .
\end{array}\right.
$$

\section{Change of variables}

For $\eta^{1}, \eta^{2} \in H^{3}(\omega)$ with

$$
\eta^{1}, \eta^{2}>-1 \text { in } \omega,
$$

we can consider the change of variables $X_{\eta^{1}, \eta^{2}}$ defined below

$$
X_{\eta^{1}, \eta^{2}}: \Omega\left(\eta^{1}\right) \longrightarrow \Omega\left(\eta^{2}\right), \quad\left(\begin{array}{l}
y_{1} \\
y_{2} \\
y_{3}
\end{array}\right) \longmapsto\left(\begin{array}{c}
y_{1} \\
y_{2} \\
\frac{1+\eta^{2}\left(y_{1}, y_{2}\right)}{1+\eta^{1}\left(y_{1}, y_{2}\right)} y_{3}
\end{array}\right) .
$$

The mapping $X_{\eta^{1}, \eta^{2}}$ is invertible of inverse $X_{\eta^{2}, \eta^{1}}$. Moreover, using the Sobolev embedding $H^{3}(\omega) \hookrightarrow C^{1}(\bar{\omega})$ and that

$$
\operatorname{det}\left(\nabla X_{\eta^{1}, \eta^{2}}\right)=\frac{1+\eta^{2}}{1+\eta^{1}}
$$

we deduce that $X_{\eta^{1}, \eta^{2}}$ is a $C^{1}$-diffeomorphism from $\Omega\left(\eta^{1}\right)$ onto $\Omega\left(\eta^{2}\right)$.

In the case $\Omega=\Omega\left(\eta^{0}\right)$ (see 2.4 ) , we set

$$
X(t, \cdot)=X_{\eta^{0}, \eta(t, \cdot)}, \quad Y(t, \cdot)=X_{\eta(t, \cdot), \eta^{0}}
$$

and in the case $\Omega=\Omega(0)$ (see $(2.5)$ ), we set

$$
X(t, \cdot)=X_{0, \eta(t, \cdot)}, \quad Y(t, \cdot)=X_{\eta(t, \cdot), 0}
$$


We have in both cases that $Y(t, \cdot)=[X(t, \cdot)]^{-1}$.

We consider the following transformation of $u$ and $p$ :

$$
u(t, y)=(\operatorname{Cof} \nabla X(t, y))^{*} U(t, X(t, y)), \quad p(t, y)=P(t, X(t, y)) \quad(t \geqslant 0, y \in \Omega) .
$$

Here, $(\operatorname{Cof} \nabla X(t, y))^{*}$ denotes the transpose of $(\operatorname{Cof} \nabla X(t, y))$. After some standard calculations (see, for instance, [21]), the system (1.3), 1.8, 1.10 can be written as

$$
\left\{\begin{array}{rl}
\partial_{t} u-\nabla \cdot \mathbb{T}(u, p)=F(u, p, \eta) & t>0, y \in \Omega, \\
\nabla \cdot u=0 & t>0, y \in \Omega, \\
\partial_{t t} \eta+A_{1} \eta+A_{2} \partial_{t} \eta=\mathbb{H}_{\eta^{0}}(u, p)+H(u, \eta) & t>0,
\end{array}\right.
$$

with the boundary conditions

$$
\left\{\begin{array}{rl}
{\left[u-\mathcal{T} \partial_{t} \eta\right]_{n_{0}}=0} & t>0, y \in \partial \Omega, \\
{\left[2 \nu D(u) n_{0}+\beta\left(u-\mathcal{T} \partial_{t} \eta\right)\right]_{\tau_{0}}=G(u, \eta)} & t>0, y \in \partial \Omega,
\end{array}\right.
$$

and with the initial conditions

$$
\left\{\begin{aligned}
u(0, \cdot)=u^{0}=U^{0} & \text { in } \Omega, \\
\eta(0, \cdot)=\eta^{0} & \text { in } \omega, \\
\partial_{t} \eta(0, \cdot)=\eta^{1} & \text { in } \omega .
\end{aligned}\right.
$$

In order to write the nonlinearities $F, H, G$, we first set

$$
(\operatorname{Cof} \nabla Y)^{*}=\left(a_{i k}\right)_{i k} .
$$

Then

$$
\begin{gathered}
F_{i}(u, p, \eta)=\sum_{k}\left(\delta_{i k}-a_{i k}(X)\right) \partial_{t} u_{k}-\sum_{l, k} a_{i k}(X) \frac{\partial u_{k}}{\partial y_{l}}(X) \partial_{t} Y_{l}(X)-\sum_{k} \partial_{t} a_{i k}(X) u_{k} \\
+\nu \sum_{j, k, l, m}\left(a_{i k}(X) \frac{\partial Y_{m}}{\partial x_{j}}(X) \frac{\partial Y_{l}}{\partial x_{j}}(X)-\delta_{i k} \delta_{m j} \delta_{j l}\right) \frac{\partial^{2} u_{k}}{\partial y_{l} \partial y_{m}} \\
+\nu \sum_{j, k, l}\left(2 \frac{\partial a_{i k}}{\partial x_{j}}(X) \frac{\partial Y_{l}}{\partial x_{j}}(X)+a_{i k}(X) \frac{\partial^{2} Y_{l}}{\partial x_{j}^{2}}(X)\right) \frac{\partial u_{k}}{\partial y_{l}}+\nu \sum_{k} \frac{\partial^{2} a_{i k}}{\partial x_{j}^{2}}(X) u_{k} \\
+\sum_{k}\left(\delta_{k i}-\frac{\partial Y_{k}}{\partial x_{i}}(X)\right) \frac{\partial p}{\partial y_{k}}-\sum_{k, l, j} a_{k l}(X) \frac{\partial a_{i j}(X)}{\partial x_{k}} u_{l} u_{j} \\
+\sum_{k, l, j, m}\left(\delta_{i j} \delta_{k l} \delta_{k m}-a_{k l}(X) a_{i j}(X) \frac{\partial Y_{m}}{\partial x_{k}}(X)\right) u_{l} \frac{\partial u_{j}}{\partial y_{m}}, \quad i=1,2,3,
\end{gathered}
$$

and

$$
\begin{aligned}
H(u, \eta)=\nu M\left[-\sum_{j, k}\left(\frac{\partial a_{3 k}}{\partial x_{j}}(X)+\frac{\partial a_{j k}}{\partial x_{3}}(X)\right) u_{k} N_{j}+\sum_{j, k, l}\left(\delta_{3 k} \delta_{j l}\left(N_{0}\right)_{j}-a_{3 k}(X) \frac{\partial Y_{l}}{\partial x_{j}}(X) N_{j}\right) \frac{\partial u_{k}}{\partial y_{l}}\right. \\
\left.+\left(\delta_{3 l} \delta_{j k}\left(N_{0}\right)_{j}-a_{j k}(X) \frac{\partial Y_{l}}{\partial x_{3}}(X) N_{j}\right) \frac{\partial u_{k}}{\partial y_{l}}\right]
\end{aligned}
$$

To define $G$, we introduce the following notations.

$$
\tau^{1}=\left(\begin{array}{c}
1 \\
0 \\
\partial_{s_{1}} \eta
\end{array}\right), \quad \tau^{2}=\left(\begin{array}{c}
0 \\
1 \\
\partial_{s_{2}} \eta
\end{array}\right)
$$




$$
\begin{aligned}
\mathcal{W}_{k}=\nu \sum_{j, m} n_{j}\left(\frac{\partial a_{k m}}{\partial x_{j}}(X) u_{m}\right. & \left.+\frac{\partial a_{j m}}{\partial x_{k}}(X) u_{m}\right)+\beta\left(\sum_{j} a_{k j}(X) u_{j}-\mathcal{T} \partial_{t} \eta \cdot e_{k}\right) \\
& +\nu \sum_{j, m, q} n_{j}\left(a_{k m}(X) \frac{\partial u_{m}}{\partial y_{q}} \frac{\partial Y_{q}}{\partial x_{j}}(X)+a_{j m}(X) \frac{\partial u_{m}}{\partial y_{q}} \frac{\partial Y_{q}}{\partial x_{k}}(X)\right), \quad k=1,2,3
\end{aligned}
$$

and

$$
\mathcal{V}^{i}=\left(2 \nu D(u) n_{0}+\beta\left(u-\mathcal{T} \partial_{t} \eta\right)\right) \cdot \tau_{0}^{i}-\mathcal{W} \cdot \tau^{i}, \quad i=1,2 .
$$

Then $G(u, \eta)$ is given by

$$
\begin{aligned}
G_{1}(u, \eta) & =\frac{\mathcal{V}^{1}\left(\left(\partial_{s_{2}} \eta^{0}\right)^{2}+1\right)-\mathcal{V}^{2}\left(\partial_{s_{1}} \eta^{0} \partial_{s_{2}} \eta^{0}\right)}{\left|N_{0}\right|^{2}}, \\
G_{2}(u, \eta) & =\frac{\mathcal{V}^{2}\left(\left(\partial_{s_{1}} \eta^{0}\right)^{2}+1\right)-\mathcal{V}^{1}\left(\partial_{s_{1}} \eta^{0} \partial_{s_{2}} \eta^{0}\right)}{\left|N_{0}\right|^{2}} \\
G_{3}(u, \eta) & =\frac{\partial_{s_{1}} \eta^{0} \mathcal{V}^{1}+\partial_{s_{2}} \eta^{0} \mathcal{V}^{2}}{\left|N_{0}\right|^{2}}
\end{aligned}
$$

More precisely, let us note that

$$
[2 \nu D(U) n+\beta(U-\mathcal{T} \partial \eta)]_{\tau}=0 \quad t>0, x \in \partial \Omega(\eta)
$$

writes as

$$
\left(2 \nu D(u) n_{0}+\beta\left(u-\mathcal{T} \partial_{t} \eta\right)\right) \cdot \tau_{0}^{i}=\mathcal{V}^{i}, \quad i=1,2 .
$$

The formula (3.14) for $G$ is such that

$$
G \cdot \tau_{0}^{i}=\mathcal{V}^{i}, \quad i=1,2, \quad G \cdot n_{0}=0
$$

so that 3.16 is equivalent to the second condition of $(3.6)$, with $G$ tangential.

Using the above transformation, we can now introduce our definition of strong solutions for system $1.3,, 1.8$, 1.10

Definition 3.1. The triplet $(U, P, \eta)$ is a strong solution of $(1.3),(1.8),(1.10)$ if the following conditions are satisfied

$$
\begin{gathered}
\eta \in W^{2}\left(0, T ; \mathcal{D}\left(A_{1}\right), L_{0}^{2}(\omega)\right), \\
1+\eta>0 \quad \text { in }[0, T],
\end{gathered}
$$

$X$ and $Y$ are given by 3.2 and $(u, p)$ are given by (3.4),

$(u, p) \in W\left(0, T ;\left[H^{2}(\Omega)\right]^{3},\left[L^{2}(\Omega)\right]^{3}\right) \times L^{2}\left(0, T ; H^{1}(\Omega) / \mathbb{R}\right)$,

$(u, p, \eta)$ satisfies the system (3.5), 3.6], 3.7].

Following this definition, in order to prove Theorem 1.1. we have to prove the existence and uniqueness of

$$
(u, p, \eta) \in W\left(0, T ;\left[H^{2}(\Omega)\right]^{3},\left[L^{2}(\Omega)\right]^{3}\right) \times L^{2}\left(0, T ; H^{1}(\Omega) / \mathbb{R}\right) \times W^{2}\left(0, T ; \mathcal{D}\left(A_{1}\right), L_{0}^{2}(\omega)\right)
$$

solution of the system (3.5), 3.6, 3.7) and satisfying (D2). 


\section{Regularity properties of the Stokes system}

In this section, we obtain some results on the stationary system in $\Omega(\eta)$ for $\eta=\eta^{0}$ (see 2.4 ) or for $\eta=0$ (see (2.5):

$$
\left\{\begin{aligned}
\alpha \bar{u}-\nu \Delta \bar{u}+\nabla \bar{p}=\bar{f} & & \text { in } \Omega(\eta), \\
\nabla \cdot \bar{u}=\bar{g} & & \text { in } \Omega(\eta), \\
\bar{u}_{n}=\bar{a} & & \text { on } \partial \Omega(\eta), \\
{[2 \nu D(\bar{u}) n+\beta \bar{u}]_{\tau}=\bar{b} } & & \text { on } \partial \Omega(\eta)
\end{aligned}\right.
$$

Let define the following space

$$
H_{\tau}^{1}=\left\{\phi \in\left[H^{1}(\Omega(\eta))\right]^{3} \mid \phi_{n}=0 \text { on } \partial \Omega(\eta)\right\}
$$

We give the definition of a weak solution of the system 4.1.

Definition 4.1. We say that $(\bar{u}, \bar{p})$ is a weak solution of 4.1 if $(\bar{u}, \bar{p}) \in\left[H^{1}(\Omega(\eta))\right]^{3} \times L^{2}(\Omega(\eta)) / \mathbb{R}$ and the following variational equation is satisfied:

$$
\alpha \int_{\Omega(\eta)} \bar{u} \cdot \phi d y+2 \nu \int_{\Omega(\eta)} D(\bar{u}): D(\phi) d y-\int_{\Omega(\eta)} \bar{p} \nabla \cdot \phi d y+\int_{\partial \Omega(\eta)} \beta v \cdot \phi d \Gamma=\int_{\Omega(\eta)} f \cdot \phi d y+\int_{\partial \Omega(\eta)} b \cdot \phi d \Gamma
$$

for all $\phi^{1} \in H_{\tau}^{1}$.

We have the following result

Theorem 4.2. Assume $\beta \geqslant 0$ and $\alpha \geqslant 0$ with $\beta_{1}+\beta_{2}>0$ or $\alpha>0$. Let $\eta \in H^{3}(\Omega(\eta))$ and $\delta_{0}>0$ such that $1+\eta>\delta_{0}$ on $\omega$. For any

$$
\bar{f} \in\left(H_{\tau}^{1}\right)^{\prime}, \quad \bar{g} \in L^{2}(\Omega(\eta)), \quad \bar{a} \in\left[H^{1 / 2}(\partial \Omega(\eta))\right]^{3}, \quad \bar{b} \in\left[H^{-1 / 2}(\partial \Omega(\eta))\right]^{3},
$$

such that

$$
\int_{\Omega(\eta)} \bar{g} d y=\int_{\partial \Omega(\eta)} \bar{a} \cdot n d \Gamma, \quad b \cdot n=0,
$$

there exists a unique weak solution $(\bar{u}, \bar{p}) \in\left[H^{1}(\Omega(\eta))\right]^{3} \times L_{0}^{2}(\Omega(\eta))$ to the Stokes system (4.1). Moreover, we have the following estimates:

$$
\|\bar{u}\|_{\left[H^{1}(\Omega(\eta))\right]^{3}}+\|\nabla \bar{p}\|_{\left[H^{-1}(\Omega(\eta))\right]^{3}} \leqslant C\left(\|\bar{f}\|_{\left(H_{\tau}^{1}\right)^{\prime}}+\|\bar{g}\|_{L^{2}(\Omega(\eta))}+\|\bar{a}\|_{\left[H^{1 / 2}(\partial \Omega(\eta))\right]^{3}}+\|\bar{b}\|_{\left[H^{-1 / 2}(\partial \Omega(\eta))\right]^{3}}\right)
$$

where $C$ is a constant which depends on $\|\eta\|_{H^{3}(\omega)}$ and $\delta_{0}$.

Moreover, if

$$
\bar{f} \in\left[L^{2}(\Omega(\eta))\right]^{3}, \quad \bar{g} \in H^{1}(\Omega(\eta)), \quad \bar{a} \in\left[H^{3 / 2}(\partial \Omega(\eta))\right]^{3}, \quad \bar{b} \in\left[H^{1 / 2}(\partial \Omega(\eta))\right]^{3},
$$

such that 4.2 holds, then $(\bar{u}, \bar{p}) \in\left[H^{2}(\Omega(\eta))\right]^{3} \times\left(H^{1}(\Omega(\eta)) \cap L_{0}^{2}(\Omega(\eta))\right)$ and we have the following estimates:

$$
\|\bar{u}\|_{\left[H^{2}(\Omega(\eta))\right]^{3}}+\|\nabla \bar{p}\|_{\left[L^{2}(\Omega(\eta))\right]^{3}} \leqslant C\left(\|\bar{f}\|_{\left[L^{2}(\Omega(\eta))\right]^{3}}+\|\bar{g}\|_{H^{1}(\Omega(\eta))}+\|\bar{a}\|_{\left[H^{3 / 2}(\partial \Omega(\eta))\right]^{3}}+\|\bar{b}\|_{\left[H^{1 / 2}(\partial \Omega(\eta))\right]^{3}}\right)
$$

where $C$ is a constant which depends on $\|\eta\|_{H^{3}(\omega)}$ and $\delta_{0}$.

In the case where $\eta \in C^{1,1}(\omega)$ such a result is already known, see [1] (see also [4]). Here, we manage to obtain the result for $\eta \in H^{3}(\omega)$ by following an idea of [14] and [15]. 
Proof of Theorem 4.2. The proof follows closely the proof of Lemma 6 in [15]. We assume here $\beta_{1}+\beta_{2}>0$, the proof is similar with $\alpha>0$.

First, we write the system 4.1 in the domain

$$
\Omega=\Omega(0)
$$

by using the change of variables $X_{0, \eta}$ defined by 3.1 . Then we set

$$
B_{\eta}=\operatorname{Cof}\left(\nabla X_{0, \eta}\right), \quad A_{\eta}=\frac{1}{\operatorname{det}\left(\nabla X_{0, \eta}\right)} B_{\eta}^{*} B_{\eta},
$$

and we define

$$
\begin{gathered}
u=\bar{u} \circ X_{0, \eta}, \quad p=\bar{p} \circ X_{0, \eta}, \\
f=\operatorname{det}\left(\nabla X_{0, \eta}\right) \bar{f} \circ X_{0, \eta}, \quad g=\operatorname{det}\left(\nabla X_{0, \eta}\right) \bar{g} \circ X_{0, \eta}, \\
a=\bar{a} \circ X_{0, \eta}, \quad b_{i}=B_{\eta}^{-1}\left(\bar{b}_{i} \circ X_{0, \eta}\right) \cdot e_{i}, \quad i=1,2 .
\end{gathered}
$$

Then system 4.1 is transformed into the following system

$$
\left\{\begin{aligned}
-\nabla \cdot\left(\nabla u A_{\eta}\right)+B_{\eta} \nabla p=f & \text { in } \Omega, \\
\nabla \cdot\left(B_{\eta}^{*} u\right)=g & \text { in } \Omega, \\
\left(B_{\eta}^{*} u\right) \cdot n_{0}=\left(B_{\eta}^{*} a\right) \cdot n_{0} & \text { on } \partial \Omega, \\
{\left[\frac{\nu}{|N|}\left(\left(B_{\eta}^{-1} \nabla u A_{\eta}\right) n_{0}+\frac{1}{\operatorname{det}\left(\nabla X_{0, \eta}\right)}\left((\nabla u)^{*} B_{\eta}\right) n_{0}\right)+\beta B_{\eta}^{-1} u\right] \cdot e_{i}=b_{i}, \quad i=1,2 } & \text { on } \partial \Omega,
\end{aligned}\right.
$$

where $N$ is defined by 1.5 and $n_{0}$ is the unit exterior normal to $\Omega$ (that is $\pm e_{3}$ ).

Since $\eta \in H^{3}(\omega)$, we deduce that

$$
B_{\eta}, A_{\eta} \in H^{2}\left(\omega ; H^{s}(0,1)\right),
$$

for all $s \geqslant 0$ and the corresponding norms depend on $\|\eta\|_{H^{3}(\omega)}$ and $\delta_{0}$. Moreover, using the embeddings $H^{1}(\omega) \hookrightarrow L^{p}(\omega)$ for all $p \geqslant 1$ and $H^{2}(\omega) \hookrightarrow L^{\infty}(\omega)$, we deduce that it is sufficient to prove that the solution of (4.6) satisfies

$$
\|u\|_{\left[H^{2}(\Omega)\right]^{3}}+\|\nabla p\|_{\left[L^{2}(\Omega)\right]^{3}} \leqslant C\left(\|f\|_{\left[L^{2}(\Omega)\right]^{3}}+\|g\|_{H^{1}(\Omega)}+\|a\|_{\left[H^{3 / 2}(\partial \Omega)\right]^{3}}+\|b\|_{\left[H^{1 / 2}(\partial \Omega)\right]^{3}}\right) .
$$

Step 1: Weak solutions. Let note that the solution of 4.6 verifies

$$
\nabla \cdot\left(\frac{1}{\operatorname{det}\left(\nabla X_{0, \eta}\right)} B_{\eta}(\nabla u)^{*} B_{\eta}\right)=B_{\eta} \nabla\left(\frac{\nabla \cdot\left(B_{\eta}^{*} u\right)}{\operatorname{det}\left(\nabla X_{0, \eta}\right)}\right)=B_{\eta} \nabla\left(\frac{g}{\operatorname{det}\left(\nabla X_{0, \eta}\right)}\right) .
$$

Let $\lambda>0$ and consider the following system

$$
\left\{\begin{aligned}
-\nabla \cdot\left(\nabla u A_{\eta}+\frac{1}{\operatorname{det}\left(\nabla X_{0, \eta}\right)} B_{\eta}(\nabla u)^{*} B_{\eta}\right)+B_{\eta} \nabla p=\tilde{f} & \text { in } \Omega, \\
\lambda p+\nabla \cdot\left(B_{\eta}^{*} u\right)=g & \text { in } \Omega, \\
\left(B_{\eta}^{*} u\right) \cdot n_{0}=\left(B_{\eta}^{*} a\right) \cdot n_{0} & \text { on } \partial \Omega, \\
{\left[\frac{\nu}{|N|}\left(\left(B_{\eta}^{-1} \nabla u A_{\eta}\right) n_{0}+\frac{1}{\operatorname{det}\left(\nabla X_{0, \eta}\right)}\left((\nabla u)^{*} B_{\eta}\right) n_{0}\right)+\beta B_{\eta}^{-1} u\right] \cdot e_{i}=b_{i}, \quad i=1,2 } & \text { on } \partial \Omega,
\end{aligned}\right.
$$

with

$$
\widetilde{f}=f-B_{\eta} \nabla\left(\frac{g}{\operatorname{det}\left(\nabla X_{0, \eta}\right)}\right)
$$


To simplify the notations, we set

$$
D_{\eta}(u)=\nabla u A_{\eta}+\frac{1}{\operatorname{det}\left(\nabla X_{0, \eta}\right)} B_{\eta}(\nabla u)^{*} B_{\eta}
$$

We define

$$
V=\left\{v \in\left[H^{1}(\Omega)\right]^{3} \mid\left(B_{\eta}^{*} v\right) \cdot n_{0}=0 \text { on } \partial \Omega\right\} .
$$

We look for weak solutions to the system 4.8 . Let $f \in V^{\prime}, g \in L^{2}(\Omega), a \in\left[H^{1 / 2}(\partial \Omega)\right]^{3}$ and $b \in\left[H^{-1 / 2}(\partial \Omega)\right]^{3}$. We have $B_{\eta} \nabla\left(\frac{g}{\operatorname{det}\left(\nabla X_{0, \eta}\right)}\right) \in V^{\prime}$ :

$$
\left\langle B_{\eta} \nabla\left(\frac{g}{\operatorname{det}\left(\nabla X_{0, \eta}\right)}\right), v\right\rangle_{V^{\prime}, V}=-\int_{\Omega} \frac{g}{\operatorname{det}\left(\nabla X_{0, \eta}\right)} \nabla \cdot\left(B_{\eta}^{*} v\right) d y .
$$

Therefore $\tilde{f} \in V^{\prime}$ and we multiply the first equation of 4.8 by $v \in V$ and the second equation of 4.8 by $\psi \in L^{2}(\Omega)$ to obtain

$$
\begin{aligned}
\int_{\Omega} D_{\eta}(u): \nabla v d y-\int_{\Omega} p \nabla \cdot\left(B_{\eta}^{*} v\right) d y+\lambda & \int_{\Omega} p \cdot \psi+\left(\nabla \cdot\left(B_{\eta}^{*} u\right)\right) \cdot \psi d y+\int_{\partial \Omega} \frac{|N|}{\nu} \beta u \cdot v d \Gamma \\
& =\langle\widetilde{f}, v\rangle_{V^{\prime}, V}+\int_{\Omega} g \cdot \psi d y+\left\langle b, \frac{|N|\left(\operatorname{det}\left(\nabla X_{0, \eta}\right)\right.}{\nu} v\right\rangle_{H^{-1 / 2}, H^{1 / 2}}
\end{aligned} .
$$

We consider a lifting $w$ satisfying

$$
\left\{\begin{aligned}
\nabla \cdot\left(B_{\eta}^{*} w\right)=g & \text { in } \Omega \\
\left(B_{\eta}^{*} w\right) \cdot n_{0}=\left(B_{\eta}^{*} a\right) \cdot n_{0} & \text { on } \partial \Omega
\end{aligned}\right.
$$

In order to this, we use [4, Corollary 8.2] and 4.2 to deduce the existence of $\bar{w} \in\left[H^{1}(\Omega)\right]^{3}$ such that

$$
\left\{\begin{aligned}
\nabla \cdot \bar{w}=g & \text { in } \Omega \\
\bar{w} \cdot n_{0}=\left(B_{\eta}^{*} a\right) \cdot n_{0} & \text { on } \partial \Omega .
\end{aligned}\right.
$$

Then $w=\left(B_{\eta}^{*}\right)^{-1} \bar{w}$ satisfies 4.10 and the estimate

$$
\|w\|_{\left[H^{1}(\Omega)\right]^{3}} \leqslant C\left(\|g\|_{L^{2}(\Omega)}+\|a\|_{\left[H^{1 / 2}(\partial \Omega)\right]^{3}}\right) .
$$

We set $u=\widehat{u}+w$. Then, a couple $(u, p)$ is a weak solution of the system 4.8 if and only if $(\widehat{u}, p)$ verifies the following variational formulation

$$
\begin{aligned}
\int_{\Omega} D_{\eta}(\widehat{u}): \nabla v d y & -\int_{\Omega} p \nabla \cdot\left(B_{\eta}^{*} v\right) d y+\lambda \int_{\Omega} p \cdot \psi+\left(\nabla \cdot\left(B_{\eta}^{*} \widehat{u}\right)\right) \cdot \psi d y+\int_{\partial \Omega} \frac{|N|}{\nu} \beta \widehat{u} \cdot v d \Gamma \\
=-\int_{\Omega} D_{\eta}(w): \nabla v d y+\langle\widetilde{f}, v\rangle_{V^{\prime}, V}+\left\langle b, \frac{|N|\left(\operatorname{det}\left(\nabla X_{0, \eta}\right)\right.}{\nu} v\right\rangle_{H^{-1 / 2}, H^{1 / 2}} & -\int_{\partial \Omega} \frac{|N|}{\nu} \beta w \cdot v d \Gamma \quad\left(v \in V, \psi \in L^{2}(\Omega)\right)
\end{aligned}
$$

We have that

$$
\int_{\Omega} D_{\eta}(v): \nabla v d y=\int_{\Omega} \frac{\left|\nabla v B_{\eta}^{*}+B_{\eta}(\nabla v)^{*}\right|^{2}}{\operatorname{det}\left(\nabla X_{0, \eta}\right)} d y
$$

and writing

$$
\bar{v}=v \circ X_{\eta, 0},
$$


we deduce

$$
\int_{\Omega} \frac{\left|\nabla v B_{\eta}^{*}+B_{\eta}(\nabla v)^{*}\right|^{2}}{\operatorname{det}\left(\nabla X_{0, \eta}\right)} d y=\int_{\Omega(\eta)}|D(\bar{v})|^{2} d x, \quad \forall v \in V,
$$

with $\bar{v} \cdot n=0$ on $\partial \Omega(\eta)$. Applying a Korn inequality (see Proposition 4.5 below):

$$
\int_{\Omega} D_{\eta}(v): \nabla v d y+\int_{\partial \Omega} \frac{|N|}{\nu} \beta|v|^{2} d \Gamma \geqslant C\|v\|_{H^{1}(\Omega)} \quad(v \in V) .
$$

Hence, we can apply the Lax-Milgram theorem and using (4.11), we deduce the existence of a unique solution of $(u, p)=\left(u_{\lambda}, p_{\lambda}\right) \in\left[H^{1}(\Omega)\right]^{3} \times L^{2}(\Omega)$ for 4.8$)$ which verifies the estimates

$$
\|u\|_{\left[H^{1}(\Omega)\right]^{3}}+\lambda\|p\|_{L^{2}(\Omega)} \leqslant C\left(\|f\|_{V^{\prime}}+\|b\|_{\left[H^{-1 / 2}(\partial \Omega)\right]^{3}}+\|g\|_{L^{2}(\Omega)}+\|a\|_{\left[H^{1 / 2}(\partial \Omega)\right]^{3}}\right) .
$$

Taking $\psi=0$ and $v \in\left[H_{0}^{1}(\Omega)\right]^{3}$ in 4.9 , we obtain

$$
\int_{\Omega} D_{\eta}(u): \nabla v d y+\int_{\Omega} \nabla p \cdot\left(B_{\eta}^{*} v\right) d y=\langle\widetilde{f}, v\rangle_{V^{\prime}, V} .
$$

This shows that $\nabla p \in\left[H^{-1}(\Omega)\right]^{3}$ and using standard result (see, for instance [4, Proposition 1.1]), we deduce

$$
\|p\|_{L^{2}(\Omega) / \mathbb{R}} \leqslant C\left(\|f\|_{V^{\prime}}+\|v\|_{\left[H^{1}(\Omega)\right]^{3}}+\|w\|_{\left[H^{1}(\Omega)\right]^{3}}\right) .
$$

Then, combining 4.15, 4.16) and (4.11), we obtain the estimate independent of $\lambda$ :

$$
\|u\|_{\left[H^{1}(\Omega)\right]^{3}}+\|p\|_{L^{2}(\Omega) / \mathbb{R}} \leqslant C\left(\|f\|_{V^{\prime}}+\|b\|_{\left[H^{-1 / 2}(\partial \Omega)\right]^{3}}+\|g\|_{L^{2}(\Omega)}+\|a\|_{\left[H^{1 / 2}(\partial \Omega)\right]^{3}}\right) .
$$

We can thus pass to the limit as $\lambda \rightarrow 0$ in 4.8 to obtain a weak solution $(u, p)$ of 4.6 . Using the above coercivity argument we also deduce the uniqueness of the weak solution of 4.6 .

Step 2: Strong solutions. We use an argument developed in [14] and [15]: if we approximate $\eta$ by $\eta_{\varepsilon} \in$ $C^{1,1}(\omega)$, and the corresponding $u_{\varepsilon}, p_{\varepsilon}$ are $H^{2}$ and $H^{1}$. We show below that their norms depend only on the $H^{3}$ norm of $\eta_{\varepsilon}$ so that we can pass to the limit. To simplify, we do not write any $\varepsilon$ below.

We first differentiate system (4.6) with respect to $y_{1}$ and $y_{2}$ to obtain a similar problem as (4.6) with source and boundary terms corresponding to the differentiates of $f, g, a$ and $b$ and to terms coming from the $A_{\eta}$ and $B_{\eta}$. We only need to estimate these terms, that is

$$
\begin{aligned}
& \left\|\nabla \cdot\left(\nabla u \partial_{y_{i}} A_{\eta}\right)-\partial_{y_{i}} B_{\eta} \nabla p\right\|_{V^{\prime}}, \quad\left\|\nabla \cdot\left(\partial_{y_{i}} B_{\eta}^{*} u\right)\right\|_{L^{2}(\Omega)}, \quad\left\|B_{\eta}^{-1} \partial_{y_{i}} B_{\eta}^{*} u\right\|_{\left[H^{1 / 2}(\partial \Omega)\right]^{3}}, \\
& \left\|\partial_{y_{i}} B_{\eta}^{-1} \nabla u A_{\eta}\right\|_{\left[H^{-1 / 2}(\partial \Omega)\right]^{9}}, \quad\left\|B_{\eta}^{-1} \nabla u \partial_{y_{i}} A_{\eta}\right\|_{\left[H^{-1 / 2}(\partial \Omega)\right]^{9}}, \\
& \left\|\partial_{y_{i}} \frac{1}{\operatorname{det}\left(\nabla X_{0, \eta}\right)}(\nabla u)^{*} B_{\eta}\right\|_{\left[H^{-1 / 2}(\partial \Omega)\right]^{9}},\left\|\frac{1}{\operatorname{det}\left(\nabla X_{0, \eta}\right)}(\nabla u)^{*} \partial_{y_{i}} B_{\eta}\right\|_{\left.H^{-1 / 2}(\partial \Omega)\right]^{9}} .
\end{aligned}
$$

Here we use a nice idea proposed in [14] and [15]: we estimate the above terms by using the $H^{2}$ regularity of $u$ and the $H^{1}$ regularity of $p$. More precisely, using the embeddings $H^{1 / 2}(\omega) \subset L^{4}(\omega)$ and $H^{1 / 4}(\omega) \subset L^{8 / 3}(\omega)$, we deduce that the above terms are estimated by

$$
\|\eta\|_{H^{3}(\omega)}\left(\|u\|_{\left[H^{1}(\Omega)\right]^{3}}^{1 / 4}\|u\|_{\left[H^{2}(\Omega)\right]^{3}}^{3 / 4}+\|p\|_{L^{2}(\Omega)}^{1 / 4}\|p\|_{H^{1}(\Omega)}^{3 / 4}\right) .
$$

Using the first part of this proof and in particular (4.17), we obtain for $i=1,2$

$$
\begin{aligned}
\left\|\partial_{y_{i}} u\right\|_{\left[H^{1}(\Omega)\right]^{3}}+\left\|\partial_{y_{i}} p\right\|_{L_{0}^{2}(\Omega)} \leqslant C\left(\|f\|_{\left[L^{2}(\Omega)\right]^{3}}+\|b\|_{\left[H^{1 / 2}(\partial \Omega)\right]^{3}}+\|g\|_{H^{1}(\Omega)}+\|a\|_{\left[H^{3 / 2}(\partial \Omega)\right]^{3}}\right) & \\
& +C\|\eta\|_{H^{3}(\omega)}\left(\|u\|_{\left[H^{1}(\Omega)\right]^{3}}^{1 / 4}\|u\|_{\left[H^{2}(\Omega)\right]^{3}}^{3 / 4}+\|p\|_{L^{2}(\Omega)}^{1 / 4}\|p\|_{H^{1}(\Omega)}^{3 / 4}\right)
\end{aligned}
$$


We differentiate $(4.6)_{2}$ with respect to $y_{3}$, we obtain

$$
\begin{array}{r}
\left\|-y_{3} \partial_{y_{1}} \eta \partial_{y_{3}}^{2} u_{1}-y_{3} \partial_{y_{2}} \eta \partial_{y_{3}}^{2} u_{2}+\partial_{y_{3}}^{2} u_{3}\right\|_{L^{2}(\Omega)} \leqslant C\left(\|f\|_{\left[L^{2}(\Omega)\right]^{3}}+\|b\|_{\left[H^{1 / 2}(\partial \Omega)\right]^{3}}+\|g\|_{H^{1}(\Omega)}+\|a\|_{\left[H^{3 / 2}(\partial \Omega)\right]^{3}}\right) \\
+C\|\eta\|_{H^{3}(\omega)}\left(\|u\|_{\left[H^{1}(\Omega)\right]^{3}}^{1 / 4}\|u\|_{\left[H^{2}(\Omega)\right]^{3}}^{3 / 4}+\|p\|_{L^{2}(\Omega)}^{1 / 4}\|p\|_{H^{1}(\Omega)}^{3 / 4}\right)
\end{array}
$$

Then, going back to 4.6$)_{1}$, we also obtain

$$
\begin{aligned}
\left\|A_{33} \partial_{y_{3}}^{2} u_{1}-y_{3} \partial_{y_{1}} \eta \partial_{y_{3}} p\right\|_{L^{2}(\Omega)}+\left\|A_{33} \partial_{y_{3}}^{2} u_{2}-y_{3} \partial_{y_{2}} \eta \partial_{y_{3}} p\right\|_{L^{2}(\Omega)}+\left\|A_{33} \partial_{y_{3}}^{2} u_{3}+\partial_{y_{3}} p\right\|_{L^{2}(\Omega)} \\
\leqslant C\left(\|f\|_{\left[L^{2}(\Omega)\right]^{3}}+\|b\|_{\left[H^{1 / 2}(\partial \Omega)\right]^{3}}+\|g\|_{H^{1}(\Omega)}+\|a\|_{\left[H^{3 / 2}(\partial \Omega)\right]^{3}}\right) \\
+C\|\eta\|_{H^{3}(\omega)}\left(\|u\|_{\left[H^{1}(\Omega)\right]^{3}}^{1 / 4}\|u\|_{\left[H^{2}(\Omega)\right]^{3}}^{3 / 4}+\|p\|_{L^{2}(\Omega)}^{1 / 4}\|p\|_{H^{1}(\Omega)}^{3 / 4}\right)
\end{aligned}
$$

Since $A_{33}=\frac{1}{1+\eta}\left(1+\left(y_{3} \partial_{y_{1}} \eta\right)^{2}+\left(y_{3} \partial_{y_{2}} \eta\right)^{2}\right)>0$, we deduce

$$
\begin{aligned}
\left\|\partial_{y_{3}}^{2} u\right\|_{L^{2}(\Omega)^{3}}+\left\|\partial_{y_{3}} p\right\|_{L^{2}(\Omega)} \leqslant C\left(\|f\|_{\left[L^{2}(\Omega)\right]^{3}}\right. & \left.+\|b\|_{\left[H^{1 / 2}(\partial \Omega)\right]^{3}}+\|g\|_{H^{1}(\Omega)}+\|a\|_{\left[H^{3 / 2}(\partial \Omega)\right]^{3}}\right) \\
& +C\|\eta\|_{H^{3}(\omega)}\left(\|u\|_{\left[H^{1}(\Omega)\right]^{3}}^{1 / 4}\|u\|_{\left[H^{2}(\Omega)\right]^{3}}^{3 / 4}+\|p\|_{L^{2}(\Omega)}^{1 / 4}\|p\|_{H^{1}(\Omega)}^{3 / 4}\right)
\end{aligned}
$$

Combining this with 4.19, we deduce the result.

We also need the following theorem which is proved in 32 .

Theorem 4.3. Assume $\beta \geqslant 0$ with $\beta_{1}+\beta_{2}>0$. Let $\eta \in H^{3}(\omega)$ and $\delta_{0}>0$ such that $1+\eta>\delta_{0}$ on $\omega$. Let us consider the following non stationary Stokes system:

$$
\left\{\begin{aligned}
\partial_{t} v-\nabla \cdot \mathbb{T}(v, \pi) & =0 & & t>0, y \in \Omega \\
\nabla \cdot v & =0 & & t>0, y \in \Omega \\
v_{n_{0}} & =0 & & t>0, y \in \partial \Omega \\
{\left[2 \nu D(v) n_{0}+\beta v\right]_{\tau_{0}} } & =\widetilde{g} & & t>0, y \in \partial \Omega \\
v(0, \cdot) & =0 & & y \in \Omega .
\end{aligned}\right.
$$

There exists $\gamma_{0}>0$ such that if

$$
\widetilde{g} \in W_{\gamma}^{1 / 4}\left(0, \infty ;\left[H^{1 / 2}(\partial \Omega)\right]^{3},\left[L^{2}(\partial \Omega)\right]^{3}\right), \quad \widetilde{g}_{n_{0}}=0 .
$$

for some $\gamma \in\left[0, \gamma_{0}\right]$. Then the problem 4.22 admits a unique solution which satisfies the estimate

$$
\|v\|_{W_{\gamma}\left(0, \infty,\left[H^{2}(\Omega)\right]^{3},\left[L^{2}(\Omega)\right]^{3}\right)}^{2}+\|\nabla \pi\|_{L_{\gamma}^{2}\left(0, \infty ;\left[L^{2}(\Omega)\right]^{3}\right)}^{2} \leqslant C\|\widetilde{g}\|_{W_{\gamma}^{1 / 4}\left(0, \infty ;\left[H^{1 / 2}(\partial \Omega)\right]^{3},\left[L^{2}(\partial \Omega)\right]^{3}\right)}^{2}
$$

where $C$ is a positive constant.

We recall that the spaces $W_{\gamma}^{s}\left(0, \infty ; X_{1}, X_{2}\right)$ and $L_{\gamma}^{2}\left(0, \infty ; L^{2}(\Omega)\right)$ are defined by $(2.3), 2.2$.

Remark 4.4. In [32], the author assumes that $\eta$ is more regular but such an assumption is only used to obtain a lift of the boundary condition by taking a stationary Stokes system of the form (4.1), see relation (75) in [32].

Note also that in [32], the condition (4.23] is replaced by the equivalent condition

$$
\widetilde{g} \in W_{\gamma}^{1 / 2}\left(0, \infty ;\left[H^{1}(\Omega)\right]^{3},\left[L^{2}(\Omega)\right]^{3}\right), \quad \widetilde{g}_{n_{0}}=0
$$

Such an equivalence can be obtained by using the surjectivity of the trace operator (see [25, p.21, Theorem 2.3]).

We end this section by proving a Korn's type inequality (that we used in the above proof). 
Proposition 4.5. Assume $\eta \in W^{1, \infty}(\omega)$. Assume that $\beta_{1}+\beta_{2} \neq 0$. There exists a positive constant $C>0$, such that

$$
\|u\|_{\left[H^{1}(\Omega(\eta))\right]^{3}} \leqslant C\left(\|D(u)\|_{\left[L^{2}(\Omega(\eta))\right]^{9}}+\|\sqrt{\beta} u\|_{\left[L^{2}(\partial \Omega(\eta))\right]^{3}}\right),
$$

for all $u \in\left[H^{1}(\Omega(\eta))\right]^{3}$.

Proof. We first show by contradiction that

$$
\|u\|_{\left[L^{2}(\Omega(\eta))\right]^{3}} \leqslant C\left(\|D(u)\|_{\left[L^{2}(\Omega(\eta))\right]^{9}}+\|\sqrt{\beta} u\|_{\left[L^{2}(\partial \Omega(\eta))\right]^{3}}\right) .
$$

Assume $u_{k} \in\left[H^{1}(\Omega(\eta))\right]^{3}$ with

$$
\left\|u_{k}\right\|_{\left[L^{2}(\Omega(\eta))\right]^{3}}=1
$$

and

$$
\left\|D\left(u_{k}\right)\right\|_{\left[L^{2}(\Omega(\eta))\right]^{9}}+\left\|\sqrt{\beta} u_{k}\right\|_{\left[L^{2}(\partial \Omega(\eta))\right]^{3}} \rightarrow 0 .
$$

Using the classical Korn inequality (see, for instance, [20]), the above relations imply that $\left(u_{k}\right)_{k}$ converges weakly to some $u \in\left[H^{1}(\Omega(\eta))\right]^{3}$ with $D(u)=0$ and $\sqrt{\beta} u=0$ on $\partial \Omega(\eta)$. In particular, see [34, Lemma 1.1 p.18], there exist $a, b \in \mathbb{R}^{3}$, such that for any $y \in \Omega(\eta), u(y)=a+b \wedge y$. Using that

$$
u\left(y+L_{1} e_{1}\right)=u(y), \quad u\left(y+L_{2} e_{2}\right)=u(y), \quad(y \in \Omega(\eta)),
$$

we deduce that $b=0$, then $u=a$ in $\Omega(\eta)$. Since $\sqrt{\beta} u=0$ on $\partial \Omega(\eta)$, we obtain that $u=0$ in $\Omega(\eta)$. Up to a subsequence $u_{k} \rightarrow u$ strongly in $\left[L^{2}(\Omega(\eta))\right]^{3}$ and thus from 4.27$)$, we get $\|u\|_{\left[L^{2}(\Omega(\eta))\right]^{3}}=1$ which leads to a contradiction. In order to prove 4.25, we combine 4.26 and the classical Korn inequality (using that $\Omega(\eta)$ is Lipschitz continuous).

\section{Linear System}

Let us consider a linearized system of 3.5, , 3.6, 3.7):

$$
\left\{\begin{array}{rl}
\partial_{t} u-\nabla \cdot \mathbb{T}(u, p)=f & t>0, y \in \Omega, \\
\nabla \cdot u=0 & t>0, y \in \Omega, \\
\partial_{t t} \eta+A_{1} \eta+A_{2} \partial_{t} \eta=-\mathcal{T}^{*}\left(\mathbb{T}(u, p) n_{0}\right)+h & t>0,
\end{array}\right.
$$

with the boundary conditions

$$
\left\{\begin{array}{rlrl}
{\left[u-\mathcal{T} \partial_{t} \eta\right]_{n_{0}}} & =0 & t>0, y \in \partial \Omega \\
{\left[2 \nu D(u) n_{0}+\beta\left(u-\mathcal{T} \partial_{t} \eta\right)\right]_{\tau_{0}}} & =\widetilde{g} & & t>0, y \in \partial \Omega
\end{array}\right.
$$

and with the initial conditions

$$
\left\{\begin{aligned}
u(0, \cdot)=u^{0} & \text { in } \Omega \\
\eta(0, \cdot)=\eta^{0} & \text { in } \omega \\
\partial_{t} \eta(0, \cdot)=\eta^{1} & \text { in } \omega .
\end{aligned}\right.
$$

Let us consider $(v, \pi)$ the solution of 4.22 associated with $\widetilde{g}$. Then $w=u-v$ and $q=p-\pi$ satisfy

$$
\left\{\begin{array}{rlrl}
\partial_{t} w-\nabla \cdot \mathbb{T}(w, q) & =f & & t>0, y \in \Omega, \\
\nabla \cdot w & =0 & & t>0, y \in \Omega \\
\partial_{t t} \eta+A_{1} \eta+A_{2} \partial_{t} \eta=-\mathcal{T}^{*}\left(\mathbb{T}(w, q) n_{0}\right)-\mathcal{T}^{*}\left(\mathbb{T}(v, \pi) n_{0}\right)+h & & t>0
\end{array}\right.
$$

with the boundary conditions

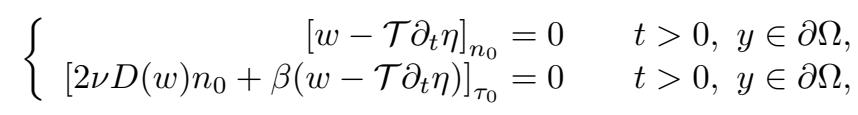


and with the initial conditions

$$
\left\{\begin{aligned}
w(0, \cdot)=u^{0} & \text { in } \Omega \\
\eta(0, \cdot)=\eta^{0} & \text { in } \omega \\
\partial_{t} \eta(0, \cdot)=\eta^{1} & \text { in } \omega
\end{aligned}\right.
$$

To solve (5.4)-(5.6), we use a semigroup approach. We endow the space $\left[L^{2}(\Omega)\right]^{3} \times \mathcal{D}\left(A_{1}^{1 / 2}\right) \times L_{0}^{2}(\omega)$ with the scalar product

$$
\left\langle\left(\begin{array}{c}
w \\
\eta_{1} \\
\eta_{2}
\end{array}\right),\left(\begin{array}{c}
v \\
\xi_{1} \\
\xi_{2}
\end{array}\right)\right\rangle=\langle w, v\rangle_{\left[L^{2}(\Omega)\right]^{3}}+\left\langle A_{1}^{1 / 2} \eta_{1}, A_{1}^{1 / 2} \xi_{1}\right\rangle_{L^{2}(\omega)}+\left\langle\eta_{2}, \xi_{2}\right\rangle_{L^{2}(\omega)}
$$

We consider the following functional spaces

$$
\begin{gathered}
\mathbb{H}=\left\{\left(w, \eta_{1}, \eta_{2}\right) \in\left[L^{2}(\Omega)\right]^{3} \times \mathcal{D}\left(A_{1}^{1 / 2}\right) \times L_{0}^{2}(\omega) \mid \nabla \cdot w=0 \quad \text { in } \Omega, \quad\left[w-\mathcal{T} \eta_{2}\right]_{n_{0}}=0 \quad \text { on } \partial \Omega\right\}, \\
\mathbb{V}=\left(\left[H^{1}(\Omega)\right]^{3} \times \mathcal{D}\left(A_{1}^{3 / 4}\right) \times \mathcal{D}\left(A_{1}^{1 / 4}\right)\right) \cap \mathbb{H} .
\end{gathered}
$$

We also denote by $\mathbb{P}$ the orthogonal projector

$$
\mathbb{P}:\left[L^{2}(\Omega)\right]^{3} \times \mathcal{D}\left(A_{1}^{1 / 2}\right) \times L_{0}^{2}(\omega) \longrightarrow \mathbb{H} .
$$

Finally, we define

$$
\begin{gathered}
\mathcal{D}(\mathcal{A})=\left\{\left(w, \eta_{1}, \eta_{2}\right) \in\left(\left[H^{2}(\Omega)\right]^{3} \times \mathcal{D}\left(A_{1}\right) \times \mathcal{D}\left(A_{1}^{1 / 2}\right)\right) \cap \mathbb{V} \mid\left[2 \nu D(w) n_{0}+\beta\left(w-\mathcal{T} \eta_{2}\right)\right]_{\tau_{0}}=0 \quad \text { on } \partial \Omega\right\} \\
\mathcal{A}\left(\begin{array}{c}
w \\
\eta_{1} \\
\eta_{2}
\end{array}\right)=\left(\begin{array}{c}
-\nu \Delta w \\
-\eta_{2} \\
A_{1} \eta_{1}+A_{2} \eta_{2}+\mathcal{T}^{*}\left(2 \nu D(w) n_{0}\right)
\end{array}\right)
\end{gathered}
$$

and

$$
\mathcal{D}(A)=\mathcal{D}(\mathcal{A}), \quad A=\mathbb{P} \mathcal{A} .
$$

Using the above definition, we can write $(5.4)-(5.6)$ as

$$
W^{\prime}+A W=\mathbb{P} F, \quad W(0)=W^{0},
$$

with

$$
W=\left(\begin{array}{c}
w \\
\eta \\
\partial_{t} \eta
\end{array}\right), \quad F=\left(\begin{array}{c}
f \\
0 \\
h
\end{array}\right)
$$

Proposition 5.1. Assume that $\beta_{1}+\beta_{2} \neq 0$. The operator A defined by $(5.8-5.10$ is the infinitesimal generator of a strongly continuous semigroup of contraction on $\mathbb{H}$.

Proof. First we show that the operator $A$ is dissipative: assume $W=\left(\begin{array}{c}w \\ \eta_{1} \\ \eta_{2}\end{array}\right) \in \mathcal{D}(A)$. Then, by integration by parts, we obtain:

$$
\langle A W, W\rangle=\langle\mathcal{A} W, W\rangle=2 \nu \int_{\Omega}|D(w)|^{2} d y-\int_{\partial \Omega} 2 \nu D(w) n_{0} \cdot\left[w-\mathcal{T}\left(\eta_{2}\right)\right] d \Gamma+\int_{\omega}\left|A_{2}^{1 / 2} \eta_{2}\right|^{2} d s .
$$

We write

$$
-\int_{\partial \Omega} 2 \nu D(w) n_{0} \cdot\left[w-\mathcal{T}\left(\eta_{2}\right)\right] d \Gamma=-\int_{\partial \Omega} 2 \nu\left[D(w) n_{0}\right]_{\tau_{0}} \cdot\left[w-\mathcal{T}\left(\eta_{2}\right)\right]_{\tau_{0}} d \Gamma=\int_{\partial \Omega} \beta\left|\left[w-\mathcal{T}\left(\eta_{2}\right)\right]_{\tau_{0}}\right|^{2} d \Gamma
$$


and we deduce

$$
\langle A W, W\rangle=2 \nu \int_{\Omega}|D(w)|^{2} d y+\int_{\omega}\left|A_{2}^{1 / 2} \eta_{2}\right|^{2} d s+\int_{\partial \Omega} \beta\left|\left[w-\mathcal{T}\left(\eta_{2}\right)\right]_{\tau_{0}}\right|^{2} d \Gamma \geqslant 0 .
$$

Second, we show that the operator $A$ is m-dissipative: we prove that for some $\lambda>0$ the operator $\lambda I+A$ is onto. Let $F=\left(\begin{array}{l}f \\ g \\ h\end{array}\right) \in \mathbb{H}$. The problem is to find $\left(\begin{array}{l}w \\ \eta_{1} \\ \eta_{2}\end{array}\right) \in \mathcal{D}(A)$ solution of the equation

$$
(\lambda I+A)\left(\begin{array}{c}
w \\
\eta_{1} \\
\eta_{2}
\end{array}\right)=F
$$

which is equivalent to the system

$$
\begin{aligned}
\lambda w-\nabla \cdot \mathbb{T}(w, q)=f & \text { in } \Omega, \\
\nabla \cdot w=0 & \text { in } \Omega, \\
\lambda \eta_{1}-\eta_{2}=g & \text { on } \omega, \\
\lambda \eta_{2}+A_{1} \eta_{1}+A_{2} \eta_{2}=-\mathcal{T}^{*}\left(\mathbb{T}(w, q) n_{0}\right)+h & \text { on } \omega, \\
{\left[w-\mathcal{T} \eta_{2}\right]_{n_{0}}=0 } & \text { on } \partial \Omega, \\
{\left[2 \nu D(w) n_{0}+\beta\left(w-\mathcal{T} \eta_{2}\right)\right]_{\tau_{0}}=0 } & \text { on } \partial \Omega .
\end{aligned}
$$

To solve the above system, we use that $\eta_{1}=\frac{1}{\lambda}\left(g+\eta_{2}\right)$ to obtain a system in $\left(u, \eta_{2}\right)$ and we introduce the space

$$
\mathcal{V}=\left\{(\phi, \xi) \in\left[H^{1}(\Omega)\right]^{3} \times \mathcal{D}\left(A_{1}^{1 / 2}\right) \mid \nabla \cdot \phi=0 \quad \text { in } \Omega, \quad[\phi-\mathcal{T} \xi]_{n_{0}}=0 \quad \text { on } \partial \Omega\right\}
$$

We can thus write the equation $\sqrt{5.12}$ in a variational form: find $\left(w, \eta_{2}\right) \in \mathcal{V}$ such that

$$
a\left(\left(\begin{array}{l}
w \\
\eta_{2}
\end{array}\right),\left(\begin{array}{l}
\phi \\
\xi
\end{array}\right)\right)=L\left(\begin{array}{l}
\phi \\
\xi
\end{array}\right) \quad\left(\left(\begin{array}{l}
\phi \\
\xi
\end{array}\right) \in \mathcal{V}\right)
$$

with $a: \mathcal{V} \times \mathcal{V} \longrightarrow \mathbb{R}$ given by

$$
\begin{aligned}
a\left(\left(\begin{array}{c}
w \\
\eta_{2}
\end{array}\right),\left(\begin{array}{l}
\phi \\
\xi
\end{array}\right)\right)=\lambda \int_{\Omega} w \cdot \phi d y+2 \nu & \int_{\Omega} D(w): D(\phi) d y+\lambda \int_{\omega} \eta_{2} \cdot \xi d s+\int_{\omega}\left(A_{2} \eta_{2}\right) \cdot \xi d s \\
& +\frac{1}{\lambda} \int_{\omega}\left(A_{1}^{1 / 2} \eta_{2}\right) \cdot\left(A_{1}^{1 / 2} \xi\right) d s+\int_{\partial \Omega} \beta\left[w-\mathcal{T}\left(\eta_{2}\right)\right]_{\tau_{0}} \cdot[\phi-\mathcal{T}(\xi)]_{\tau_{0}} d \Gamma
\end{aligned}
$$

and $L: \mathcal{V} \longrightarrow \mathbb{R}$ given by

$$
L\left(\begin{array}{l}
\phi \\
\xi
\end{array}\right)=\int_{\Omega} f \cdot \phi d y+\int_{\omega} h \cdot \xi d s-\frac{1}{\lambda} \int_{\omega}\left(A_{1}^{1 / 2} g\right) \cdot\left(A_{1}^{1 / 2} \xi\right) d s .
$$

The bilinear form $a$ is continuous and coercive on $\mathcal{V}$ thanks to the classical Korn inequality. We can also check that $L$ is linear and continuous on $\mathcal{V}$. By the Lax-Milgram theorem, there exists a unique $\left(u, \eta_{2}\right) \in \mathcal{V}$ solution of 5.14 .

Now, taking $\xi=0$ and $\phi \in \mathcal{D}_{\sigma}(\Omega)$, the equation 5.14 becomes

$$
\lambda \int_{\Omega} w \cdot \phi d y+2 \nu \int_{\Omega} D(w): D(\phi) d y=\int_{\Omega} f \cdot \phi d y
$$

which is equivalent to

$$
\langle\lambda w-\nu \Delta w-f, \phi\rangle=0, \quad \forall \phi \in \mathcal{D}_{\sigma}(\Omega)
$$


Using the De Rham theorem [33, Proposition 1.2, p.14] , we deduce the existence of a unique $q \in L^{2}(\Omega) / \mathbb{R}$ such that (5.13a) holds. In particular, we have $\nabla \cdot \mathbb{T}(w, q) \in\left[L^{2}(\Omega)\right]^{3}$ and $\mathbb{T}(w, q) \in\left[L^{2}(\Omega)\right]^{9}$. Therefore, we deduce that $\mathbb{T}(w, q) n_{0} \in\left[H^{-1 / 2}(\partial \Omega)\right]^{3}$ and

$$
\int_{\Omega} \mathbb{T}(w, q): D(\phi) d y-\left\langle\mathbb{T}(w, q) n_{0}, \phi\right\rangle_{H^{-1 / 2}, H^{1 / 2}}=\int_{\Omega}(f-\lambda w) \cdot \phi d y
$$

for all $\phi \in\left[H^{1}(\Omega)\right]^{3}, \nabla \cdot \phi=0, \phi_{n_{0}}=0$. On the other hand, taking $\xi=0$ in (5.14) yields

$$
\lambda \int_{\Omega} w \cdot \phi d y+2 \nu \int_{\Omega} D(w): D(\phi) d y+\left\langle\beta\left[w-\mathcal{T}\left(\eta_{2}\right)\right]_{\tau_{0}}, \phi\right\rangle_{H^{-1 / 2}, H^{1 / 2}}=\int_{\Omega} f \cdot \phi d y,
$$

for all $\phi \in\left[H^{1}(\Omega)\right]^{3}, \nabla \cdot \phi=0, \phi_{n_{0}}=0$. Comparing (5.15) and (5.16) and taking into account that

$$
\int_{\Omega} \mathbb{T}(w, q): D(\phi) d y=2 \nu \int_{\Omega} D(w): D(\phi) d y, \quad \forall \phi \in\left[H^{1}(\Omega)\right]^{3}, \nabla \cdot \phi=0, \phi_{n_{0}}=0,
$$

we obtain

$$
-\left\langle\mathbb{T}(w, q) n_{0}, \phi\right\rangle_{H^{-1 / 2}, H^{1 / 2}}=\left\langle\left[\beta\left(w-\mathcal{T} \eta_{2}\right)\right]_{\tau_{0}}, \phi\right\rangle_{H^{-1 / 2}, H^{1 / 2}}=0, \quad \forall \phi \in\left[H^{1}(\Omega)\right]^{3}, \nabla \cdot \phi=0, \phi_{n_{0}}=0 .
$$

Let $\phi \in\left[H^{1 / 2}(\partial \Omega)\right]^{3}$ such that $\phi_{n_{0}}=0$, and let consider the system

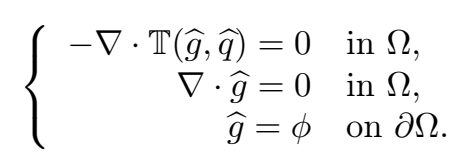

The above system admits a unique solution $(\widehat{g}, \widehat{q}) \in\left[H^{1}(\Omega)\right]^{3} \times L_{0}^{2}(\Omega)$ such that $\nabla \cdot \widehat{g}=0$ and $\left.\widehat{g}\right|_{\partial \Omega}=\phi$. This implies that (5.17) holds for all $\phi \in\left[H^{1}(\Omega)\right]^{3}, \phi_{n_{0}}=0$. Inserting (5.17) in 5.15) we get

$$
\int_{\Omega} 2 \nu D(w): D(\phi) d y-\int_{\Omega} q \nabla \cdot \phi d y+\left\langle\beta\left(w-\mathcal{T} \eta_{2}\right)_{\tau_{0}}, \phi_{\tau_{0}}\right\rangle_{H^{-1 / 2}, H^{1 / 2}}=\int_{\Omega}(f-\lambda w) \cdot \phi d y,
$$

for all $\phi \in\left[H^{1}(\Omega)\right]^{3}, \phi_{n_{0}}=0$.

Thus, we deduce that $(w, q)$ is a weak solution of (5.13a), (5.13b), (5.13e) and (5.13f) in the sense of Definition 4.1. Since $\eta_{2} \in H^{2}(\omega), \mathcal{T} \eta_{2} \in\left[H^{2}(\partial \Omega)\right]^{3}$ we can apply Theorem 4.2 and obtain $(w, q) \in\left[H^{2}(\Omega)\right]^{3} \times H^{1}(\Omega) / \mathbb{R}$.

Going back to the variational formulation (5.14), we deduce

$$
\int_{\omega}\left(A_{1}^{1 / 2} \eta_{1}\right) \cdot\left(A_{1}^{1 / 2} \xi\right) d s=-\lambda \int_{\omega} \eta_{2} \cdot \xi d s-\int_{\omega}\left(A_{2} \eta_{2}\right) \cdot \xi d s-\int_{\omega} \mathcal{T}^{*}\left(\mathbb{T}(u, q) n_{0}\right) \cdot \xi d s+\int_{\omega} h \cdot \xi d s
$$

for any $\xi \in \mathcal{D}\left(A_{1}^{1 / 2}\right)$ and where $\eta_{1}=\frac{1}{\lambda}\left(g+\eta_{2}\right)$. We have $\mathbb{T}(w, q) n_{0} \in\left[H^{1 / 2}(\partial \Omega)\right]^{3}$ and thus $\mathcal{T}^{*}\left(\mathbb{T}(w, q) n_{0}\right) \in$ $L_{0}^{2}(\omega)$. Moreover since $\eta_{2} \in H^{2}(\omega)$, we deduce that $\eta_{2} \in \mathcal{D}\left(A_{2}\right)$. Thus $A_{1} \eta_{1} \in L_{0}^{2}(\omega)$.

Applying Lumer-Phillips theorem, we conclude that $\left(e^{-t A}\right)_{t \geqslant 0}$ is a semigroup of contractions on $\mathbb{H}$.

In order to prove that $\left(e^{-t A}\right)_{t \geqslant 0}$ is an analytical semigroup, we use Lemma 3.10 in [2]. We first need to show that $\left(e^{-t A}\right)_{t \geqslant 0}$ is exponentially stable.

Proposition 5.2. Assume that $\beta_{1}+\beta_{2} \neq 0$. The semigroup $\left(e^{-t A}\right)_{t \geqslant 0}$ is exponentially stable.

Proof. Since $\left(e^{-t A}\right)_{t \geqslant 0}$ is a semigroup of contraction, we apply the classical result of Huang-Gearhart (see for instance [26, Theorem 1.3.2, p.4]). We have to show that

$$
i \mathbb{R} \subset \rho(A) \text { and } \sup _{\lambda \in \mathbb{R}}\left\|(i \lambda I+A)^{-1}\right\|<\infty .
$$


Using the proof of [2, Proposition 3.5], we only need to prove the existence of $C>0$ such that

$$
\forall \lambda \in \mathbb{C}, \quad \operatorname{Re} \lambda \in(0,1), \quad\left\|(\lambda I+A)^{-1}\right\|_{\mathbb{H}} \leqslant C .
$$

Let us consider $\lambda \in \mathbb{C}$, with $\operatorname{Re} \lambda \in(0,1), F=\left(\begin{array}{l}f \\ g \\ h\end{array}\right) \in \mathbb{H}$ and $\left(\begin{array}{l}w \\ \eta_{1} \\ \eta_{2}\end{array}\right) \in \mathcal{D}(A)$ such that

$$
(\lambda I+A)\left(\begin{array}{c}
w \\
\eta_{1} \\
\eta_{2}
\end{array}\right)=F .
$$

We can write the above relation as the system (5.13). We multiply (5.13a) by $\bar{w}, 5.13 \mathrm{~d}$ ) by $\bar{\eta}_{2}$ and we perfom integrations by parts to deduce

$$
\begin{aligned}
& \operatorname{Re} \lambda\left(\|w\|_{\left[L^{2}(\Omega)\right]^{3}}^{2}+\left\|\eta_{2}\right\|_{L^{2}(\omega)}^{2}+\left\|A_{1}^{1 / 2} \eta_{1}\right\|_{L^{2}(\omega)}^{2}\right)+2 \nu\|D w\|_{\left[L^{2}(\Omega)\right]^{9}}^{2}+\int_{\partial \Omega} \beta\left|\left(w-\mathcal{T} \eta_{2}\right)_{\tau_{0}}\right|^{2} d \Gamma \\
&+\left\|A_{2}^{1 / 2} \eta_{2}\right\|_{L^{2}(\omega)}^{2} \leqslant C\|F\|_{\mathbb{H}}\left\|\left(w, \eta_{1}, \eta_{2}\right)\right\|_{\mathbb{H}}
\end{aligned}
$$

We have

$$
\left\|\eta_{2}\right\|_{L_{0}^{2}(\omega)}^{2} \leqslant C\left\|A_{2}^{1 / 2} \eta_{2}\right\|_{L_{0}^{2}(\omega)}^{2} \leqslant C\|F\|_{\mathbb{H}}\left\|\left(w, \eta_{1}, \eta_{2}\right)\right\|_{\mathbb{H}} .
$$

On the other hand, we have

$$
\|w\|_{\left[L^{2}(\partial \Omega)\right]^{3}}^{2} \leqslant C\left(\left\|\beta\left(w-\mathcal{T} \eta_{2}\right)\right\|_{\left[L^{2}(\partial \Omega)\right]^{3}}^{2}+\left\|\mathcal{T} \eta_{2}\right\|_{\left[L^{2}(\partial \Omega)\right]^{3}}^{2}\right) .
$$

Using 4.25, 5.21 and the fact that $\mathcal{T} \in \mathcal{L}\left(L^{2}(\omega),\left[L^{2}(\partial \Omega)\right]^{3}\right)$, we obtain

$$
\|w\|_{\left[H^{1}(\Omega)\right]^{3}}^{2} \leqslant C\|F\|_{\mathbb{H}}\|W\|_{\mathbb{H}} \cdot
$$

Following the proof of Proposition 3.5 in [2], we have

$$
\left\|A_{1}^{1 / 2} \eta_{1}\right\|_{L_{0}^{2}(\omega)}^{2} \leqslant C\left(\|w\|_{H^{1}(\Omega)}^{2}+\|F\|_{\mathbb{H}}^{2}+\|F\|_{\mathbb{H}}\left\|\left(w, \eta_{1}, \eta_{2}\right)\right\|_{\mathbb{H}}\right) .
$$

Gathering the above inequality with 5.22 and 5.21 , we obtain

$$
\left\|\left(w, \eta_{1}, \eta_{2}\right)\right\|_{\mathbb{H}} \leqslant C\|F\|_{\mathbb{H}},
$$

for some positive constant $C$. This concludes the proof.

Proposition 5.3. Suppose that $\beta_{1}+\beta_{2} \neq 0$. The operator $A$ is the infinitesimal generator of an analytic semigroup on $\mathbb{H}$.

Proof. We apply Lemma 3.10 in [2]: since $\left(e^{-t A}\right)_{t \geqslant 0}$ is exponentially stable, it sufficient to show

$$
\left\|(\lambda I+A)^{-1} F\right\|_{\mathbb{H}} \leqslant \frac{C}{|\lambda|}\|F\|_{\mathbb{H}} \quad\left(F \in \mathbb{H}, \lambda \in i \mathbb{R}^{*}\right) .
$$

Assume $\lambda \in i \mathbb{R}^{*}, F=\left(\begin{array}{l}f \\ g \\ h\end{array}\right) \in \mathbb{H}$ and let us consider $W=(\lambda I+A)^{-1} F$. We write $W=\left(\begin{array}{c}w \\ \eta_{1} \\ \eta_{2}\end{array}\right)$ so that (5.13) holds. We now proceed as in [2, Proposition 3.11]: we multiply (5.13a by $\bar{u}$ and (5.13d) by $\bar{\eta}_{2}$ and we integrate 
by parts

$$
\begin{aligned}
\lambda\left(\int_{\Omega}|w|^{2} d y+\left\|\eta_{2}\right\|_{L^{2}(\omega)}^{2}-\left\|A_{1}^{1 / 2} \eta_{1}\right\|_{L^{2}(\omega)}^{2}\right)+2 \nu \int_{\Omega}|D w|^{2} d y & +\left\|A_{2}^{1 / 2} \eta_{2}\right\|_{L^{2}(\omega)}^{2} \\
& +\int_{\partial \Omega} \beta\left|\left(w-\mathcal{T} \eta_{2}\right)_{\tau_{0}}\right|^{2} d \Gamma=\langle F, W\rangle .
\end{aligned}
$$

Multiplying by $\bar{\lambda}$ and taking the real part, we find

$$
|\lambda|^{2}\|W\|_{\mathbb{H}}^{2}=2|\lambda|^{2}\left\|A_{1}^{1 / 2} \eta_{1}\right\|_{L^{2}(\omega)}^{2}+\operatorname{Re}\langle F ; \lambda W\rangle
$$

Using the Cauchy-Schwarz inequality, we obtain

$$
|\lambda|^{2}\|W\|_{\mathbb{H}}^{2} \leqslant 4|\lambda|^{2}\left\|A_{1}^{1 / 2} \eta_{1}\right\|_{L^{2}}^{2}+\|F\|_{\mathbb{H}}^{2} .
$$

Since $A_{1}$ and $A_{2}$ are self-adjoint positive operators and $\mathcal{D}\left(A_{1}^{1 / 4}\right)=\mathcal{D}\left(A_{2}^{1 / 2}\right)$, we apply [11, Theorem 1.1] to deduce that

$$
\mathbb{A}=\left[\begin{array}{cc}
0 & I \\
A_{1} & A_{2}
\end{array}\right]
$$

is the infinitesimal generator of an analytical semigroup on $\mathcal{D}\left(A_{1}^{1 / 2}\right) \times L_{0}^{2}(\omega)$. We have in particular

$$
|\lambda|\left\|(\lambda I+\mathbb{A})^{-1} Z\right\|_{\mathcal{D}\left(A_{1}^{1 / 2}\right) \times L_{0}^{2}(\omega)} \leqslant C\|Z\|_{\mathcal{D}\left(A_{1}^{1 / 2}\right) \times L_{0}^{2}(\omega)} \quad\left(\lambda \in i \mathbb{R}^{*}, Z \in \mathcal{D}\left(A_{1}^{1 / 2}\right) \times L_{0}^{2}(\omega)\right) .
$$

Applying this estimate on $5.13 \mathrm{c}-5.13 \mathrm{~d}$, we deduce

$$
|\lambda|\left(\left\|A_{1}^{1 / 2} \eta_{1}\right\|_{L^{2}(\omega)}+\left\|\eta_{2}\right\|_{L^{2}(\omega)}\right) \leqslant C\left(\left\|\mathcal{T}^{*}\left(\mathbb{T}(w, q) n_{0}\right)\right\|_{L^{2}(\omega)}+\left\|A_{1}^{1 / 2} g\right\|_{L^{2}(\omega)}+\|h\|_{L^{2}(\omega)}\right) .
$$

We use the fact $\mathcal{T}^{*} \in \mathcal{L}\left(\left[L^{2}(\partial \Omega)\right]^{3}, L_{0}^{2}(\omega)\right)$ and we combine 5.26 and 5.25 to find

$$
\left.|\lambda|\|W\|_{\mathbb{H}} \leqslant C\left(\| \mathbb{T}(w, q) n_{0}\right)\left\|_{\left[L^{2}(\partial \Omega)\right]^{3}}+\right\| F \|_{\mathbb{H}}\right) .
$$

Combining Theorem 4.2 and an interpolation argument, we get for $\varepsilon<1 / 4$

$$
\left\|\mathbb{T}(w, q) n_{0}\right\|_{\left[L^{2}(\partial \Omega)\right]^{3}} \leqslant C\left(\|(\nabla \cdot(\mathbb{T}(w, q)))\|_{\left[H^{-2 \varepsilon}(\Omega)\right]^{3}}+\left\|\mathcal{T} \eta_{2}\right\|_{\left[H^{2-2 \varepsilon}(\partial \Omega)\right]^{3}}\right) .
$$

The rest of the proof is similar to the proof of [2, Proposition 3.11].

We recall that $\mathcal{X}_{\infty, \gamma}$ is the space given in 2.8 . We are now in position to give the following theorem.

Theorem 5.4. Suppose that $\beta_{1}+\beta_{2} \neq 0$. There exists $\gamma_{0}>0$ such that if

$$
\left(u^{0}, \eta^{0}, \eta^{1}\right) \in \mathbb{V}, \quad f \in L_{\gamma}^{2}\left(0,+\infty ;\left[L^{2}(\Omega)\right]^{3}\right), \quad h \in L_{\gamma}^{2}\left(0,+\infty ; L_{0}^{2}(\omega)\right),
$$

and

$$
\widetilde{g} \in W_{\gamma}^{1 / 4}\left(0,+\infty ;\left[H^{1 / 2}(\partial \Omega)\right]^{3},\left[L^{2}(\partial \Omega)\right]^{3}\right) \quad \text { with } \quad \widetilde{g}_{n_{0}}=0
$$

for $\gamma \in\left[0, \gamma_{0}\right]$, then there exists a unique solution $(u, p, \eta) \in \mathcal{X}_{\infty, \gamma}$ on $(0,+\infty)$ of the system (5.1)-(5.3). Moreover there exists a positive constant $C$ such that

$$
\begin{array}{r}
\|(u, p, \eta)\|_{\mathcal{X}_{\infty, \gamma}} \leqslant C\left(\left\|\left(u^{0}, \eta^{0}, \eta^{1}\right)\right\|_{\mathbb{V}}+\|f\|_{L_{\gamma}^{2}\left(0,+\infty ;\left[L^{2}(\Omega)\right]^{3}\right)}+\|\widetilde{g}\|_{W_{\gamma}^{1 / 4}\left(0,+\infty ;\left[H^{1 / 2}(\partial \Omega)\right]^{3},\left[L^{2}(\partial \Omega)\right]^{3}\right)}\right. \\
\left.+\|h\|_{L_{\gamma}^{2}\left(0,+\infty ; L^{2}(\omega)\right)}\right)
\end{array}
$$


Proof. Since $A$ generates an analytical and exponentially stable semigroup, from [5, Theorem 3.1, p.143], the evolution equation (5.11) admits a unique strong solution and verifies the estimates

$$
\begin{aligned}
& \left\|\left(w, \eta_{1}, \eta_{2}\right)\right\|_{L_{\gamma}^{2}(0,+\infty ; \mathcal{D}(A))}+\left\|\left(w, \eta_{1}, \eta_{2}\right)\right\|_{L_{\gamma}^{\infty}(0,+\infty ; \mathbb{V})}+\left\|\left(w, \eta_{1}, \eta_{2}\right)\right\|_{H_{\gamma}^{1}(0,+\infty ; \mathbb{H})} \\
& \quad \leqslant C\left(\left\|\left(u^{0}, \eta^{0}, \eta^{1}\right)\right\|_{\mathbb{V}}+\|f\|_{L_{\gamma}^{2}\left(0,+\infty ;\left[L^{2}(\Omega)\right]^{3}\right)}+\|h\|_{L_{\gamma}^{2}\left(0,+\infty ; L^{2}(\omega)\right)}\right) .
\end{aligned}
$$

Applying the De Rham theorem [33, Proposition 1.2, p.14], we deduce the existence of $q \in L_{\gamma}^{2}\left(0, \infty ; H^{1}(\Omega) / \mathbb{R}\right)$ such that $(w, \eta, q)$ is the solution of (5.4)-(5.6). Setting $u=w+v, p=q+\pi$ where $(v, \pi)$ is the solution of (4.22) associated with $\widetilde{g}$, we obtain the result.

Corollary 5.5. Suppose that $\beta_{1}+\beta_{2} \neq 0$. Assume $T>0$ and

$$
\begin{gathered}
\left(u^{0}, \eta^{0}, \eta^{1}\right) \in \mathbb{V}, \quad f \in L^{2}\left(0, T ;\left[L^{2}(\Omega)\right]^{3}\right), \quad h \in L^{2}\left(0, T ; L_{0}^{2}(\omega)\right), \\
\widetilde{g} \in W^{1 / 4}\left(0, T ;\left[H^{1 / 2}(\partial \Omega)\right]^{3},\left[L^{2}(\partial \Omega)\right]^{3}\right) \quad \text { with } \quad \widetilde{g}_{n_{0}}=0 .
\end{gathered}
$$

Then there exists a unique solution $(u, p, \eta) \in \mathcal{X}_{T}$ on $(0, T)$ of the system (5.1)-(5.3). Moreover, there exists a positive constant independent of $T$ such that

$$
\begin{gathered}
\|(u, p, \eta)\|_{\mathcal{X}_{T}} \leqslant C\left(\left\|\left(u^{0}, \eta^{0}, \eta^{1}\right)\right\|_{\mathbb{V}}+\|f\|_{L^{2}\left(0, T ;\left[L^{2}(\Omega)\right]^{3}\right)}+\|\widetilde{g}\|_{W^{1 / 4}\left(0, T ;\left[H^{1 / 2}(\partial \Omega)\right]^{3},\left[L^{2}(\partial \Omega)\right]^{3}\right)}\right. \\
\left.+\|h\|_{L^{2}\left(0, T ; L^{2}(\omega)\right)}\right) .
\end{gathered}
$$

Proof. We extend $f, \widetilde{g}, h$ by 0 in $(T, \infty)$ and apply Theorem 5.4

We can now deal with the case $\beta_{i}=0$ for $i=1,2$

Theorem 5.6. Suppose that $\beta_{1}=\beta_{2}=0$. Assume $T>0$ and

$$
\begin{gathered}
\left(u^{0}, \eta^{0}, \eta^{1}\right) \in \mathbb{V}, \quad f \in L^{2}\left(0, T ;\left[L^{2}(\Omega)\right]^{3}\right), \quad h \in L^{2}\left(0, T ; L_{0}^{2}(\omega)\right), \\
\widetilde{g} \in W^{1 / 4}\left(0, T ;\left[H^{1 / 2}(\partial \Omega)\right]^{3},\left[L^{2}(\partial \Omega)\right]^{3}\right) \quad \text { with } \quad \widetilde{g}_{n_{0}}=0 .
\end{gathered}
$$

Then there exists a unique solution $(u, p, \eta) \in \mathcal{X}_{T}$ on $(0, T)$ of the system (5.1)-(5.3). Moreover, there exists a positive constant (non decreasing with respect to $T$ ) such that

$$
\begin{aligned}
\|(u, p, \eta)\|_{\mathcal{X}_{T}} \leqslant C\left(\left\|\left(u^{0}, \eta^{0}, \eta^{1}\right)\right\|_{\mathbb{V}}+\|f\|_{L^{2}\left(0, T ;\left[L^{2}(\Omega)\right]^{3}\right)}+\|\widetilde{g}\|_{W^{1 / 4}\left(0, T ;\left[H^{1 / 2}(\partial \Omega)\right]^{3},\left[L^{2}(\partial \Omega)\right]^{3}\right)}\right. \\
\left.+\|h\|_{L^{2}\left(0, T ; L^{2}(\omega)\right)}\right) .
\end{aligned}
$$

Proof. Let introduce the space

$$
\mathbb{X}=W^{1 / 4}\left(0, T ;\left[H^{1 / 2}(\partial \Omega)\right]^{3},\left[L^{2}(\partial \Omega)\right]^{3}\right) \times W^{1 / 4}\left(0, T ; H^{1 / 2}(\omega), L^{2}(\omega)\right) .
$$

Let $\left(\widetilde{u}, \widetilde{\eta_{2}}\right) \in \mathbb{X}$. From Corollary 5.5 (with $\beta_{1}=\beta_{2}=1$ ), there exists a unique strong solution $(u, p, \eta) \in \mathcal{X}_{T}$ to the system (5.1), 5.3) with the boundary conditions

$$
\left\{\begin{array}{rl}
{\left[u-\mathcal{T} \partial_{t} \eta\right]_{n_{0}}=0} & t \in(0, T), y \in \partial \Omega, \\
{\left[2 \nu D(u) n_{0}\right]_{\tau_{0}}+\left[u-\mathcal{T} \partial_{t} \eta\right]_{\tau_{0}}=\widetilde{g}+\left[\widetilde{u}-\mathcal{T} \widetilde{\eta}_{2}\right]_{\tau_{0}}} & t \in(0, T), y \in \partial \Omega .
\end{array}\right.
$$

Using the trace theorems and the definition 2.6) of $\mathcal{X}_{T}$ we can thus define the mapping

$$
\mathbb{F}: \mathbb{X} \longrightarrow \mathbb{X}, \quad\left(\begin{array}{c}
\widetilde{u} \\
\widetilde{\eta}_{2}
\end{array}\right) \longmapsto\left(\begin{array}{c}
u \\
\partial_{t} \eta
\end{array}\right) .
$$


Let us prove that the mapping $\mathbb{F}$ is a contraction for $T$ small enough: assume $\left(\widetilde{u}^{i}, \widetilde{\eta}_{2}^{i}\right) \in \mathbb{X}, i=1,2$ and let $\left(u^{i}, p^{i}, \eta^{i}\right) \in \mathcal{X}_{T} i=1,2$ be the corresponding solutions of the system 5.17, 5.3. 5.33). We write

$$
u=u^{1}-u^{2}, \quad p=p^{1}-p^{2}, \quad \eta=\eta^{1}-\eta^{2}, \quad \widetilde{u}=\widetilde{u}^{1}-\widetilde{u}^{2}, \quad \widetilde{\eta}_{2}=\widetilde{\eta}_{2}^{1}-\widetilde{\eta}_{2}^{2}
$$

so that

$$
\begin{gathered}
\left\{\begin{array}{rl}
\partial_{t} u-\nabla \cdot \mathbb{T}(u, p)=0 & t>0, y \in \Omega, \\
\nabla \cdot u=0 & t>0, y \in \Omega, \\
\partial_{t t} \eta+A_{1} \eta+A_{2} \partial_{t} \eta=-\mathcal{T}^{*}\left(\mathbb{T}(u, p) n_{0}\right) & t>0,
\end{array}\right. \\
\left\{\begin{array}{rl}
{\left[u-\mathcal{T} \partial_{t} \eta\right]_{n_{0}}=0} & t>0, y \in \partial \Omega, \\
{\left[2 \nu D(u) n_{0}+\left(u-\mathcal{T} \partial_{t} \eta\right)\right]_{\tau_{0}}=\left[\left(\widetilde{u}-\mathcal{T} \widetilde{\eta}_{2}\right)\right]_{\tau_{0}}} & t>0, y \in \partial \Omega,
\end{array}\right. \\
\left\{\begin{aligned}
u(0, \cdot)=0 & \text { in } \Omega, \\
\eta(0, \cdot)=0 & \text { in } \omega, \\
\partial_{t} \eta(0, \cdot)=0 & \text { in } \omega .
\end{aligned}\right.
\end{gathered}
$$

From (5.31) and the boundedness of $\mathcal{T}$, we obtain

$$
\|(u, p, \eta)\|_{\mathcal{X}_{T}} \leqslant C\left\|\left(\widetilde{u}, \widetilde{\eta_{2}}\right)\right\|_{\mathbb{X}} \cdot
$$

From (2.6), (2.7), the trace theorem and Lemma A.5 in [6], there exists a constant $C$ independent of $T$ such that

$$
\left\|\partial_{t} \eta\right\|_{H^{3 / 4}\left(0, T ; H^{1 / 2}(\omega)\right)}+\|v\|_{H^{5 / 8}\left(0, T ;\left[L^{2}(\partial \Omega)\right]^{3}\right)}+\|v\|_{L^{\infty}\left(0, T ;\left[H^{1 / 2}(\partial \Omega)\right]^{3}\right)} \leqslant C\|(u, p, \eta)\|_{\mathcal{X}_{T}} .
$$

From Corollary A.3 in [6] and (5.36), we deduce

$$
\left\|\partial_{t} \eta\right\|_{H^{1 / 4}\left(0, T ; L^{2}(\omega)\right)}+\|v\|_{H^{1 / 4}\left(0, T ;\left[L^{2}(\partial \Omega)\right]^{3}\right)} \leqslant C\left(T^{3 / 4}+T^{3 / 8}\right)\|(u, p, \eta)\|_{\mathcal{X}_{T}}
$$

and

$$
\left\|\partial_{t} \eta\right\|_{L^{2}\left(0, T ; H^{1 / 2}(\omega)\right)}+\|v\|_{L^{2}\left(0, T ;\left[H^{1 / 2}(\partial \Omega)\right]^{3}\right)} \leqslant C T^{1 / 2}\|(u, p, \eta)\|_{\mathcal{X}_{T}} .
$$

Combining the estimates (5.38), 5.39), 5.40), we obtain

$$
\left\|\mathbb{F}\left(\widetilde{u}^{1}, \widetilde{\eta}^{1}\right)-\mathbb{F}\left(\widetilde{u}^{2}, \widetilde{\eta}^{2}\right)\right\|_{\mathbb{X}} \leqslant C\left(T^{3 / 4}+T^{3 / 8}\right)\left\|\left(\widetilde{u}^{1}, \widetilde{\eta}^{1}\right)-\left(\widetilde{u}^{2}, \widetilde{\eta}^{2}\right)\right\|_{\mathbb{X}}
$$

This shows that $\mathbb{F}$ is a contraction for $T$ small enough and using the Banach fixed-point theorem, we deduce the existence and the uniqueness of a strong solution for the system (5.1)-(5.3) (with $\beta_{1}=\beta_{2}=0$ ) and the estimate [5.32). To deduce the result fo any $T$, we simply reiterate the above procedure on small intervals $\left[k T_{0},(k+1) T_{0}\right]$, where $T_{0}$ is such that $\mathbb{F}$ is a contraction.

\section{$6 \quad$ Fixed point}

In this section, we prove the main result Theorem 1.1. Using Definition 3.1. we first restate this result after change of variables.

\section{Theorem 6.1.}

1. Let $\beta_{i} \geqslant 0, i=1,2$. Assume that $\left(u^{0}, \eta^{0}, \eta^{1}\right) \in \mathbb{V}$ with

$$
1+\eta^{0}>0
$$

There exists a time $T_{0}>0$ (depending only on $\left\|\left(u^{0}, \eta^{0}, \eta^{1}\right)\right\|_{\mathbb{V}}$ ) such that the system (3.5), (3.6) and (3.7) admits a unique strong solution $(u, p, \eta) \in \mathcal{X}_{T}$ for $T<T_{0}$. 
2. Let $\beta_{i} \geqslant 0$ with $\beta_{1}+\beta_{2}>0, i=1,2$. There exists $R_{0}>0$ such that for any $\left(u^{0}, \eta^{0}, \eta^{1}\right) \in \mathbb{V}$ with

$$
1+\eta^{0}>0 \text { and with }\left\|\left(u^{0}, \eta^{0}, \eta^{1}\right)\right\|_{\mathbb{V}} \leqslant R_{0},
$$

then the system (3.5), 3.6 and (3.7) admits a unique strong solution $(u, p, \eta) \in \mathcal{X}_{\infty, \gamma}$ on $(0, \infty)$ for $\gamma \in\left[0, \gamma_{0}\right]$.

We recall that $\mathbb{V}$ is defined by (5.7). The above result is obtained by using a fixed-point argument.

First let us show the local in time existence. We define for all $T>0$ the space

$$
\mathcal{Y}_{T}=L^{2}\left(0, T ;\left[L^{2}(\Omega)\right]^{3}\right) \times W^{1 / 4}\left(0, T ;\left[H^{1 / 2}(\partial \Omega)\right]^{3},\left[L^{2}(\partial \Omega)\right]^{3}\right) \times L^{2}\left(0, T ; L^{2}(\omega)\right),
$$

and for $R>0$, we define the set

$$
\mathcal{B}_{T, R}=\left\{(f, \tilde{g}, h) \in \mathcal{Y}_{T} \mid\|(f, \tilde{g}, h)\|_{\mathcal{Y}_{T}} \leqslant R\right\} .
$$

In the sequel, we denote by $C$ a quantity which does not depend on $R$ and $T$. We first start by assuming

$$
\left\|\left(u^{0}, \eta^{0}, \eta^{1}\right)\right\|_{\mathbb{V}} \leqslant R .
$$

Thus, applying Theorem 5.6 we know that for any $(f, \widetilde{g}, h) \in \mathcal{B}_{T, R}$, there exists a unique solution $(u, p, \eta) \in \mathcal{X}_{T}$ of (5.1)-(5.3). Moreover, the estimate (5.29) yields

$$
\|(u, p, \eta)\|_{\mathcal{X}_{T}} \leqslant C R,
$$

for some positive constant $C$. For the local existence, the constant $R$ is fixed. In the next section, we show that for $T$ small enough, we can define $F, G, H$ by (3.9), 3.10) and (3.14) and thus consider the mapping $\Phi$ defined as follows:

$$
\Phi: \mathcal{B}_{T, R} \longrightarrow \mathcal{Y}_{T}, \quad(f, \widetilde{g}, h) \longmapsto(F(u, p, \eta), G(u, \eta), H(u, \eta)) .
$$

In what follows, we show that for $T$ small enough, we have $\Phi\left(\mathcal{B}_{T, R}\right) \subset \mathcal{B}_{T, R}$ and that $\Phi_{\mid \mathcal{B}_{T, R}}$ is a strict contraction.

First, we notice that (6.4) yields several other useful estimates. From (2.6), 2.7) and Lemma A.5 in [6], there exists a constant $C$ independent of $T$ such that

$$
\begin{gathered}
\|\eta\|_{H^{1}\left(0, T ; H^{2}(\omega)\right)}+\|\eta\|_{H^{3 / 4}\left(0, T ; H^{5 / 2}(\omega)\right)}+\left\|\partial_{t} \eta\right\|_{L^{4}\left(0, T ; H^{3 / 2}(\omega)\right)}+\left\|\partial_{s_{j}} \eta\right\|_{H^{7 / 8}\left(0, T ; H^{5 / 4}(\omega)\right)} \\
+\left\|\partial_{s_{j} s_{k}}^{2} \eta\right\|_{H^{7 / 8}\left(0, T ; L^{8 / 3}(\omega)\right)}+\|u\|_{L^{3}\left(0, T ;\left[H^{5 / 3}(\Omega)\right]^{3}\right)} \\
+\|u\|_{H^{1 / 4}\left(0, T ;\left[H^{1}(\partial \Omega)\right]^{3}\right)}+\|u\|_{H^{3 / 4}\left(0, T ;\left[L^{2}(\partial \Omega)\right]^{3}\right)} \leqslant C R .
\end{gathered}
$$

For simplicity, in all what follows, we assume

$$
T \leqslant 1 .
$$

The above assumption simplifies the estimates in the sense that we only keep the smaller power of $T$. We also denote by $C_{R}$ a constant that can depend on $R$ in a nondecreasing way (typically the sum of $C R^{m}, m \in \mathbb{N}$, $C>0)$. The value of these constants may change from one appearance to another.

\subsection{Estimates on the change of variables}

We first prove some useful estimates on $\eta$

Lemma 6.2. We have

$$
\left\|\eta-\eta^{0}\right\|_{L^{\infty}\left(0, T ; L^{\infty}(\omega)\right)} \leqslant C\left\|\eta-\eta^{0}\right\|_{L^{\infty}\left(0, T ; H^{2}(\omega)\right)} \leqslant C_{R} T^{1 / 2} .
$$


In particular, there exists

$$
T_{0}=\frac{C}{R^{2}}>0
$$

such that if $T \leqslant T_{0}$, then

$$
\left\|\frac{1}{1+\eta}\right\|_{L^{\infty}\left(0, T ; L^{\infty}(\omega)\right)} \leqslant C .
$$

We also have the following estimates

$$
\begin{gathered}
\left\|\partial_{s_{j}} \eta-\partial_{s_{j}} \eta^{0}\right\|_{L^{\infty}\left(0, T ; L^{\infty}(\omega)\right)} \leqslant C_{R} T^{1 / 4} \\
\left\|\eta-\eta^{0}\right\|_{L^{\infty}\left(0, T ; H^{5 / 2}(\omega)\right)}+\left\|\partial_{s_{j} s_{k}}^{2} \eta-\partial_{s_{j} s_{k}}^{2} \eta^{0}\right\|_{L^{\infty}\left(0, T ; L^{4}(\omega)\right)} \leqslant C_{R} T^{1 / 4} \\
\left\|\partial_{t} \eta\right\|_{L^{6}\left(0, T ; H^{1}(\omega)\right)} \leqslant C_{R} T^{1 / 6} .
\end{gathered}
$$

Proof. In order to prove $(6.8)$, we write

$$
\eta(t, \cdot)=\eta^{0}+\int_{0}^{t} \partial_{t} \eta\left(t^{\prime}, \cdot\right) d t^{\prime}
$$

and we combine it with 6.6 and with $H^{2}(\omega) \hookrightarrow L^{\infty}(\omega)$.

Since

$$
\eta^{0} \in \mathcal{D}\left(A_{1}^{3 / 4}\right)=H^{3}(\omega) \hookrightarrow C^{0}(\bar{\omega}),
$$

there exists $\varepsilon>0$ such that $1+\eta^{0}>2 \varepsilon$. Using $(6.8)$, we obtain $(6.9)$ if $T$ is small enough.

We set $\xi=\partial_{s_{j}} \eta-\partial_{s_{j}} \eta^{0}$ and $\xi^{*}\left(t^{*}, \cdot\right)=\xi\left(t^{*} T, \cdot\right), t^{*} \in[0,1]$. Then we combine A.1, the embedding $H^{3 / 4}(0,1) \hookrightarrow L^{\infty}(0,1)$, Lemma A.1 in [6] and (6.6) to obtain

$$
\begin{array}{r}
\|\xi\|_{L^{\infty}\left(0, T ; H^{3 / 2}(\omega)\right)}=\left\|\xi^{*}\right\|_{L^{\infty}\left(0,1 ; H^{3 / 2}(\omega)\right)} \leqslant C\left\|\xi^{*}\right\|_{H^{3 / 4}\left(0,1 ; H^{3 / 2}(\omega)\right)} \leqslant C\left\lfloor\xi^{*}\right\rfloor_{\left.3 / 4,2,(0,1), H^{3 / 2}(\omega)\right)} \\
=C T^{1 / 4}\lfloor\xi\rfloor_{\left.3 / 4,2,(0, T), H^{3 / 2}(\omega)\right)} \leqslant C T^{1 / 4}\left\|\partial_{s_{j}} \eta\right\|_{H^{3 / 4}\left(0, T ; H^{3 / 2}(\omega)\right)} \leqslant C T^{1 / 4} R .
\end{array}
$$

Then, we deduce (6.10) and (6.11) by using $H^{3 / 2}(\omega) \hookrightarrow L^{\infty}(\omega)$ and $H^{1 / 2}(\omega) \hookrightarrow L^{4}(\omega)$.

Finally, 6.12 is a consequence of 6.6 and 2.7.

Now, we show some estimates on the changes of variables $X$ and $Y$ defined by 3.2 . We recall that $a_{i k}$ is given by 3.8 .

Lemma 6.3. Assume 6.7.

$$
\begin{gathered}
\left\|a_{i k}(X)-\delta_{i k}\right\|_{L^{\infty}\left(0, T ; L^{\infty}(\Omega)\right)}+\left\|\nabla Y(X)-I_{3}\right\|_{\left.L^{\infty}\left(0, T ; L^{\infty}(\Omega)\right]^{9}\right)} \leqslant C_{R} T^{1 / 4} . \\
\left\|a_{i k}(X)\right\|_{L^{\infty}\left(0, T ; L^{\infty}(\Omega)\right)}+\|\nabla Y(X)\|_{L^{\infty}\left(0, T ;\left[L^{\infty}(\Omega)\right]^{9}\right)} \leqslant C_{R} . \\
\left\|\frac{\partial a_{i k}}{\partial y_{j}}(X)\right\|_{L^{\infty}\left(0, T ; L^{4}(\Omega)\right)}+\left\|\frac{\partial^{2} Y_{i}}{\partial x_{j} \partial x_{k}}(X)\right\|_{L^{\infty}\left(0, T ; L^{4}(\Omega)\right)} \leqslant C_{R} T^{1 / 4} . \\
\left\|\frac{\partial^{2} a_{i k}}{\partial x_{j}^{2}}(X)\right\|_{L^{\infty}\left(0, T ; L^{2}(\Omega)\right)} \leqslant C_{R} . \\
\left\|\partial_{t} Y(X)\right\|_{L^{4}\left(0, T ;\left[L^{\infty}(\Omega)\right]^{3}\right)} \leqslant C_{R} . \\
\left\|\partial_{t} a_{i k}(X)\right\|_{L^{6}\left(0, T ; L^{2}(\Omega)\right)} \leqslant C_{R} T^{1 / 6} .
\end{gathered}
$$


Proof. By definition (see (3.1) and (3.2), we recall that

$$
Y_{3}(t, x)=\frac{1+\eta^{0}\left(x_{1}, x_{2}\right)}{1+\eta\left(t, x_{1}, x_{2}\right)} x_{3}, \quad Y_{i}(t, x)=x_{i}, i=1,2 .
$$

As a consequence, the estimate on $\nabla Y(X)-I_{3}$ reduces to the estimate of the following terms

$$
\left\|\frac{\partial Y_{3}}{\partial x_{j}}(X)\right\|_{L^{\infty}\left(0, T ; L^{\infty}(\Omega)\right)}, \quad j=1,2 \quad \text { and }\left\|\frac{\partial Y_{3}}{\partial x_{3}}(X)-1\right\|_{L^{\infty}\left(0, T ; L^{\infty}(\Omega)\right)} .
$$

We have

$$
\frac{\partial Y_{3}}{\partial x_{3}}(X)-1=\frac{\eta^{0}-\eta}{1+\eta}
$$

By using 6.8 and 6.9, we deduce

$$
\left\|\frac{\partial Y_{3}}{\partial x_{3}}(X)-1\right\|_{L^{\infty}\left(0, T ; L^{\infty}(\Omega)\right)} \leqslant C_{R} T^{1 / 2}
$$

On the other hand, for $j=1,2$, we have

$$
\frac{\partial Y_{3}}{\partial x_{j}}(X)=y_{3} \frac{\left(\partial_{s_{j}} \eta^{0}-\partial_{s_{j}} \eta\right)}{1+\eta^{0}}+y_{3} \partial_{s_{j}} \eta \frac{\left(\eta-\eta^{0}\right)}{(1+\eta)\left(1+\eta^{0}\right)}
$$

and thus, using 6.4, 6.3, 6.8 and 6.10,

$$
\left\|\frac{\partial Y_{3}}{\partial x_{j}}(X)\right\|_{L^{\infty}\left(0, T ; L^{\infty}(\Omega)\right)} \leqslant C T^{1 / 4} R+C T^{1 / 2} R^{2} \leqslant C_{R} T^{1 / 4} .
$$

Hence, we obtain 6.14 and thus 6.15.

We have for $k, j \in\{1,2\}$,

$$
\begin{aligned}
& \frac{\partial^{2} Y_{3}}{\partial x_{k} \partial x_{j}}(X)=y_{3} \frac{\left(\partial_{s_{j} s_{k}}^{2} \eta^{0}-\partial_{s_{j} s_{k}}^{2} \eta\right)}{\left(1+\eta^{0}\right)}+y_{3} \partial_{s_{k}} \eta \frac{\left(\partial_{s_{j}} \eta-\partial_{s_{j}} \eta^{0}\right)}{(1+\eta)\left(1+\eta^{0}\right)}+y_{3} \partial_{s_{j}} \eta \frac{\left(\partial_{s_{k}} \eta-\partial_{s_{k}} \eta^{0}\right)}{(1+\eta)\left(1+\eta^{0}\right)} \\
&+y_{3}\left(\eta-\eta^{0}\right)\left(\frac{\partial_{s_{k} s_{j}} \eta}{\left(1+\eta^{0}\right)(1+\eta)}-2 \frac{\partial_{s_{k}} \eta \partial_{s_{j}} \eta}{\left(1+\eta^{0}\right)(1+\eta)^{2}}\right)
\end{aligned}
$$

Then, we obtain

$$
\begin{aligned}
\left\|\frac{\partial^{2} Y_{3}}{\partial x_{k} \partial x_{j}}(X)\right\|_{L^{\infty}\left(0, T ; L^{4}(\omega)\right)} \leqslant C\left(\left\|\partial_{s_{j} s_{k}}^{2} \eta-\partial_{s_{j} s_{k}}^{2} \eta^{0}\right\|_{L^{\infty}\left(0, T ; L^{4}(\omega)\right)}+R\left\|\partial_{s_{j}} \eta^{0}-\partial_{s_{j}} \eta\right\|_{L^{\infty}\left(0, T ; L^{\infty}(\omega)\right)}\right. \\
\left.+\left\|\eta^{0}-\eta\right\|_{L^{\infty}\left(0, T ; L^{\infty}(\omega)\right)}\left(\left\|\partial_{s_{j} s_{k}}^{2} \eta\right\|_{L^{\infty}\left(0, T ; L^{4}(\omega)\right)}+R^{2}\right)\right)
\end{aligned}
$$

Using 6.11, 6.10 and 6.8, we obtain 6.16. The other cases for $k, j$ are easier to do and we skip them. The third derivative $\frac{\partial^{3} Y}{\partial x_{j} \partial_{k} \partial x_{l}}$ involves the following terms

$$
\begin{aligned}
& y_{3} \frac{\partial_{s_{j} s_{k} s_{l}}^{3} \eta^{0}}{1+\eta^{0}}, \quad y_{3} \frac{\partial_{s_{l}} \eta \partial_{s_{j} s_{k}}^{2} \eta^{0}}{(1+\eta)\left(1+\eta^{0}\right)}, \quad y_{3} \frac{\partial_{s_{l}} \eta^{0} \partial_{s_{j} s_{k}}^{2} \eta}{(1+\eta)\left(1+\eta^{0}\right)}, \quad y_{3} \frac{\partial_{s_{j} s_{k} s_{l}}^{3} \eta}{1+\eta}, \quad y_{3} \frac{\partial_{s_{l}} \eta \partial_{s_{j} s_{k}}^{2} \eta}{(1+\eta)^{2}} \\
& y_{3} \frac{\partial_{s_{j}} \eta \partial_{s_{k}} \eta \partial_{s_{l}} \eta}{(1+\eta)^{3}}, \quad y_{3} \frac{\partial_{s_{j}} \eta \partial_{s_{k}} \eta \partial_{s_{l}} \eta^{0}}{(1+\eta)^{2}\left(1+\eta^{0}\right)}
\end{aligned}
$$


Thus, using 6.4, 6.10), 6.11, 6.8 and 2.7), we obtain 6.17).

We have

$$
\partial_{t} Y(X)=-y_{3} \frac{\partial_{t} \eta}{1+\eta} e_{3}
$$

and thus

$$
\left\|\partial_{t} Y(X)\right\|_{L^{4}\left(0, T ;\left[L^{\infty}(\Omega)\right]^{3}\right)} \leqslant C_{R}\left\|\partial_{t} \eta\right\|_{L^{4}\left(0, T ; L^{\infty}(\omega)\right)} .
$$

Thus, using (6.3) and 6.6), we obtain 6.18).

The terms appearing in $\partial_{t} a_{i k}(X)$ are of the form

$$
y_{3} \frac{\partial_{t} \eta \partial_{s_{j}} \eta}{(1+\eta)^{2}}, \quad y_{3} \frac{\partial_{t} \eta \partial_{s_{j}} \eta^{0}}{(1+\eta)\left(1+\eta^{0}\right)}, \quad y_{3} \frac{\partial_{t s_{j}}^{2} \eta}{(1+\eta)}, \quad-\frac{\left(1+\eta^{0}\right) \partial_{t} \eta}{(1+\eta)^{2}} .
$$

Consequently, using 6.8 and 6.10,

$$
\left\|\partial_{t} a_{i k}(X)\right\|_{L^{6}\left(0, T ; L^{2}(\Omega)\right)} \leqslant C_{R}\left\|\partial_{t} \eta\right\|_{L^{6}\left(0, T ; H^{1}(\omega)\right)} .
$$

The above estimate and 6.12 yield 6.19).

Now, we need the following lemma to estimates the terms on the boundary.

Lemma 6.4. Assume 6.7. Then we have the following estimates

$$
\begin{aligned}
\left\|\nabla Y(X)-I_{3}\right\|_{L^{\infty}\left(0, T ;\left[H^{3 / 2}(\partial \Omega)\right]^{9}\right)} & \left\|a_{i k}(X)-\delta_{i k}\right\|_{L^{\infty}\left(0, T ; H^{3 / 2}(\partial \Omega)\right)} \\
+ & \left\|n_{0}-n\right\|_{L^{\infty}\left(0, T ;\left[H^{3 / 2}(\partial \Omega)\right]^{3}\right)}+\left\|\tau_{0}^{i}-\tau^{i}\right\|_{L^{\infty}\left(0, T ;\left[H^{3 / 2}(\partial \Omega)\right]^{3}\right)} \leqslant C_{R} T^{1 / 4} . \\
& \left\|\frac{\partial a_{m k}}{\partial x_{j}}(X)\right\|_{L^{\infty}\left(0, T ; H^{1 / 2}(\partial \Omega)\right)} \leqslant C_{R} T^{1 / 4} . \\
\left\|\nabla Y(X)-I_{3}\right\|_{H^{7 / 8}\left(0, T ;\left[L^{\infty}(\partial \Omega)\right]^{9}\right)}+ & \left\|a_{i k}(X)-\delta_{i k}\right\|_{H^{7 / 8}\left(0, T ; L^{\infty}(\partial \Omega)\right)} \\
& +\left\|n_{0}-n\right\|_{H^{7 / 8}\left(0, T ;\left[L^{\infty}(\partial \Omega)\right]^{3}\right)}+\left\|\tau_{0}^{i}-\tau^{i}\right\|_{H^{7 / 8}\left(0, T ;\left[L^{\infty}(\partial \Omega)\right]^{3}\right)} \leqslant C_{R} . \\
& \left\|\frac{\partial a_{m k}}{\partial x_{j}}(X)\right\|_{H^{7 / 8}\left(0, T ; L^{8 / 3}(\partial \Omega)\right)} \leqslant C_{R} .
\end{aligned}
$$

Proof. Relation 6.25 is a consequence of 6.21, 6.23, 1.5 and 6.11 combined with 6.11. We obtain 6.26 by using Lemma 6.2 with (3.8).

Using (6.6) and $H^{5 / 4}(\omega) \hookrightarrow L^{\infty}(\omega)$, we obtain

$$
\left\|\partial_{s_{j}} \eta^{0}-\partial_{s_{j}} \eta\right\|_{H^{7 / 8}\left(0, T ; L^{\infty}(\omega)\right)} \leqslant C_{R} .
$$

For $\left(\alpha_{1}, \alpha_{2}, \alpha_{3}\right) \in \mathbb{N}^{3}$, we also deduce that

$$
\frac{\eta^{\alpha_{1}}\left(\partial_{s_{j}} \eta\right)^{\alpha_{2}}}{(1+\eta)^{\alpha_{3}}}\left(\partial_{s_{j}} \eta^{0}-\partial_{s_{j}} \eta\right) \in H^{7 / 8}\left(0, T ; L^{\infty}(\omega)\right) .
$$

Nevertheless, one has to take care about the dependence in $T$ of the corresponding norm. In order to do this, we notice that if

$$
f, g \in H^{7 / 8}\left(0, T ; L^{\infty}(\omega)\right) \cap L^{\infty}\left(0, T ; L^{\infty}(\omega)\right),
$$

then

$$
f g \in H^{7 / 8}\left(0, T ; L^{\infty}(\omega)\right) \cap L^{\infty}\left(0, T ; L^{\infty}(\omega)\right),
$$


and

$\|f g\|_{H^{7 / 8}\left(0, T ; L^{\infty}(\omega)\right) \cap L^{\infty}\left(0, T ; L^{\infty}(\omega)\right)} \leqslant C\|f\|_{H^{7 / 8}\left(0, T ; L^{\infty}(\omega)\right) \cap L^{\infty}\left(0, T ; L^{\infty}(\omega)\right)}\|g\|_{H^{7 / 8}\left(0, T ; L^{\infty}(\omega)\right) \cap L^{\infty}\left(0, T ; L^{\infty}(\omega)\right)}$.

The last estimate is obtained by writing the definition 2.1) of the norm in $H^{7 / 8}\left(0, T ; L^{\infty}(\omega)\right)$.

Then, combining (6.29) with 6.4 , we obtain that

$$
\left\|\frac{\eta^{\alpha_{1}}\left(\partial_{s_{j}} \eta\right)^{\alpha_{2}}}{(1+\eta)^{\alpha_{3}}}\left(\partial_{s_{j}} \eta^{0}-\partial_{s_{j}} \eta\right)\right\|_{H^{7 / 8}\left(0, T ; L^{\infty}(\omega)\right)} \leqslant C_{R} .
$$

From this estimate and 6.21), 6.23, 1.5 and (3.11), we obtain 6.27).

To prove 6.28, we use that the terms appearing in $\frac{\partial a_{m k}}{\partial x_{j}}(X)$ are of the form 6.24. Combining the above arguments with 6.6 and 6.4, we deduce the result.

\subsection{Estimates of $F, G, H$}

Proposition 6.5. Assume F, G, H are given by (3.9), 3.14, 3.10). Then we have

$$
\begin{gathered}
\|F(u, p, \eta)\|_{L^{2}\left(0, T ;\left[L^{2}(\Omega)\right]^{3}\right)} \leqslant C_{R} T^{1 / 6}, \\
\|H(u, \eta)\|_{L^{2}\left(0, T ; L^{2}(\omega)\right)} \leqslant C_{R} T^{1 / 4}, \\
\|G(u, \eta)\|_{L^{2}\left(0, T ; H^{1 / 2}(\partial \Omega)\right)}+\|G(u, \eta)\|_{H^{1 / 4}\left(0, T ; L^{2}(\partial \Omega)\right)} \leqslant C_{R} T^{1 / 8} .
\end{gathered}
$$

Proof. Using 6.14, 6.15), we obtain

$$
\begin{gathered}
\left\|\left(a_{i k}(X) \frac{\partial Y_{m}}{\partial x_{j}}(X) \frac{\partial Y_{l}}{\partial x_{j}}(X)-\delta_{i k} \delta_{m j} \delta_{j l}\right) \frac{\partial^{2} u_{k}}{\partial y_{l} \partial y_{m}}\right\|_{L^{2}\left(0, T ; L^{2}(\Omega)\right)} \leqslant C_{R} T^{1 / 4}, \\
\left\|\left(\delta_{i k}-a_{i k}(X)\right) \partial_{t} u_{k}\right\|_{L^{2}\left(0, T ; L^{2}(\Omega)\right.} \leqslant C_{R} T^{1 / 4}
\end{gathered}
$$

and

$$
\left\|\left(\delta_{k i}-\frac{\partial Y_{k}}{\partial x_{i}}(X)\right) \frac{\partial p}{\partial y_{k}}\right\|_{L^{2}\left(0, T ; L^{2}(\Omega)\right)} \leqslant C_{R} T^{1 / 4} .
$$

Using (6.15) and (6.18), we obtain

$$
\left\|a_{i k}(X) \partial_{t} Y_{l}(X) \frac{\partial u_{k}}{\partial y_{l}}\right\|_{L^{2}\left(0, T ; L^{2}(\Omega)\right)} \leqslant C_{R} T^{1 / 4}\left\|\partial_{t} Y(X)\right\|_{L^{4}\left(0, T ;\left[L^{\infty}(\Omega)\right]^{3}\right)}\|u\|_{L^{\infty}\left(0, T ;\left[H^{1}(\Omega)\right]^{3}\right)} \leqslant C_{R} T^{1 / 4} .
$$

Using 6.15) and 6.16, we get

$$
\begin{gathered}
\left\|a_{i k}(X) \frac{\partial^{2} Y_{l}}{\partial x_{j}^{2}}(X) \frac{\partial u_{k}}{\partial y_{l}}\right\|_{L^{2}\left(0, T ; L^{2}(\Omega)\right)}+\left\|\frac{\partial a_{i k}}{\partial x_{j}}(X) \frac{\partial Y_{l}}{\partial x_{j}}(X) \frac{\partial u_{k}}{\partial y_{l}}\right\|_{L^{2}\left(0, T ; L^{2}(\Omega)\right)} \\
\leqslant C_{R}\left(\left\|\frac{\partial a_{i k}}{\partial y_{j}}(X)\right\|_{L^{\infty}\left(0, T ; L^{4}(\Omega)\right)}+\left\|\frac{\partial^{2} Y_{l}}{\partial x_{j}^{2}}(X)\right\|_{L^{\infty}\left(0, T ; L^{4}(\Omega)\right)}\right)\|u\|_{L^{2}\left(0, T ;\left[H^{2}(\Omega)\right]^{3}\right)} \leqslant C_{R} T^{1 / 4}
\end{gathered}
$$

From 6.19 and 6.6 , it follows that

$$
\left\|\partial_{t} a_{i k}(X) u_{k}\right\|_{L^{2}\left(0, T ; L^{2}(\Omega)\right)} \leqslant\left\|\partial_{t} a_{i k}(X)\right\|_{L^{6}\left(0, T ; L^{2}(\Omega)\right)}\left\|u_{k}\right\|_{L^{3}\left(0, T ; L^{\infty}(\Omega)\right)} \leqslant C_{R} T^{1 / 6} .
$$


From 6.17 and 6.6

$$
\left\|\frac{\partial^{2} a_{i k}}{\partial x_{j}^{2}}(X) u_{k}\right\|_{L^{2}\left(0, T ; L^{2}(\Omega)\right)} \leqslant T^{1 / 6}\left\|\frac{\partial^{2} a_{i k}}{\partial x_{j}^{2}}(X)\right\|_{L^{\infty}\left(0, T ; L^{2}(\Omega)\right)}\left\|u_{k}\right\|_{L^{3}\left(0, T ; L^{\infty}(\Omega)\right)} \leqslant C_{R} T^{1 / 6} .
$$

Using standard estimates on the nonlinear terms (see, for instance, [3, p.48]), we have

$$
\left\|u_{l} \frac{\partial u_{j}}{\partial y_{m}}\right\|_{L^{2}\left(0, T ; L^{2}(\Omega)\right)} \leqslant C T^{1 / 4} R^{2}
$$

Combining this with 6.14 yields

$$
\left\|\left(\delta_{i j} \delta_{k l} \delta_{k m}-a_{k l}(X) a_{i j}(X) \frac{\partial Y_{m}}{\partial x_{k}}(X)\right) u_{l} \frac{\partial u_{j}}{\partial y_{m}}\right\|_{L^{2}\left(0, T ; L^{2}(\Omega)\right)} \leqslant C_{R} T^{1 / 2} .
$$

Using (6.16), we have also

$$
\begin{aligned}
& \left\|a_{k l}(X) \frac{\partial a_{i j}(X)}{\partial x_{k}} u_{l} u_{j}\right\|_{L^{2}\left(0, T ; L^{2}(\Omega)\right)} \leqslant C_{R}\left\|\frac{\partial a_{i j}}{\partial x_{k}}(X)\right\|_{L^{\infty}\left(0, T ; L^{4}(\Omega)\right)}\left\|u_{l}\right\|_{L^{\infty}\left(0, T ; L^{4}(\Omega)\right)}\left\|u_{j}\right\|_{L^{2}\left(0, T ; L^{\infty}(\Omega)\right)} \\
& \leqslant C_{R} T^{1 / 4} \text {. }
\end{aligned}
$$

Hence, $F(u, p, \eta)$ is $L^{2}\left(0, T ;\left[L^{2}(\Omega)\right]^{3}\right)$ and using 6.33, 6.34, 6.35, 6.39, 6.37) and 6.40, we get

$$
\|F(u, p, \eta)\|_{L^{2}\left(0, T ;\left[L^{2}(\Omega)\right]^{3}\right)} \leqslant C_{R} T^{1 / 6} .
$$

We estimate now $G(u, \eta)$ in $W^{1 / 4}\left(0, T ;\left[H^{1 / 2}(\partial \Omega)\right]^{3},\left[L^{2}(\partial \Omega)\right]^{3}\right)$. We recall that the formula (3.14) for $G$ involves $\tau^{i}, \mathcal{W}, \mathcal{V}^{i}$ (see (3.11), (3.12), (3.13)). First we write for $i=1,2$

$$
\mathcal{V}^{i}=\left(2 \nu D(u) n_{0}+\beta\left(u-\mathcal{T} \partial_{t} \eta\right)\right) \cdot\left(\tau_{0}^{i}-\tau^{i}\right)+\left[2 \nu D(u) n_{0}+\beta\left(u-\mathcal{T} \partial_{t} \eta\right)-\mathcal{W}\right] \cdot \tau^{i},
$$

with

$$
\begin{aligned}
& {\left[2 \nu D(u) n_{0}+\beta\left(u-\mathcal{T} \partial_{t} \eta\right)-\mathcal{W}\right]_{k}=\nu \sum_{j, m, q}\left(n_{0}\right)_{j}\left(\delta_{k m} \frac{\partial u_{m}}{\partial y_{q}} \delta_{q j}+\delta_{j m} \frac{\partial u_{m}}{\partial y_{q}} \delta_{q k}\right)} \\
& \quad-\nu \sum_{j, m, q} n_{j}\left(a_{k m}(X) \frac{\partial u_{m}}{\partial y_{q}} \frac{\partial Y_{q}}{\partial x_{j}}(X)+a_{j m}(X) \frac{\partial u_{m}}{\partial y_{q}} \frac{\partial Y_{q}}{\partial x_{k}}(X)\right) \\
& -\nu \sum_{j, m} n_{j}\left(\frac{\partial a_{k m}}{\partial x_{j}}(X) u_{m}+\frac{\partial a_{j m}}{\partial x_{k}}(X) u_{m}\right)+\beta \sum_{j}\left(\delta_{k j}-a_{k j}(X)\right) u_{j}, \quad k=1,2,3
\end{aligned}
$$

From 6.4 and trace results, we have

$$
\|u\|_{L^{2}\left(0, T ;\left[H^{3 / 2}(\partial \Omega)\right]^{3}\right)}+\left\|\frac{\partial u_{m}}{\partial y_{q}}\right\|_{L^{2}\left(0, T ;\left[H^{1 / 2}(\partial \Omega)\right]^{3}\right)} \leqslant C R .
$$

Combining this with 6.25 and 6.26 , we deduce

$$
\left\|\mathcal{V}^{i}\right\|_{L^{2}\left(0, T ; H^{1 / 2}(\partial \Omega)\right)} \leqslant C_{R} T^{1 / 4}
$$

and thus from (3.14, we finally obtain

$$
\|G(u, \eta)\|_{L^{2}\left(0, T ;\left[H^{1 / 2}(\partial \Omega)\right]^{3}\right)} \leqslant C_{R} T^{1 / 4} .
$$


For the estimate in $H^{1 / 4}\left(0, T ; L^{2}(\partial \Omega)\right)$, we use $\mathrm{A.5}$ : for instance,

$$
\begin{aligned}
\| n_{j}\left(a_{k m}(X)\right. & \left.-\delta_{k m}\right) \frac{\partial u_{m}}{\partial y_{q}} \frac{\partial Y_{q}}{\partial x_{j}}(X) \|_{H^{1 / 4}\left(0, T ; L^{2}(\partial \Omega)\right)} \\
& \leqslant C T^{1 / 8}\left\|n_{j}\left(a_{k m}(X)-\delta_{k m}\right) \frac{\partial Y_{q}}{\partial x_{j}}(X)\right\|_{H^{7 / 8}\left(0, T ; L^{2}(\partial \Omega)\right)}\left\|\frac{\partial u_{m}}{\partial y_{q}}\right\|_{H^{1 / 4}\left(0, T ; L^{2}(\partial \Omega)\right)} \leqslant C_{R} T^{1 / 8},
\end{aligned}
$$

The last inequality is obtained by using both 6.25), 6.27) and (6.6).

The other kind of terms that has to be estimated are of the form

$$
\left\|\frac{\partial a_{k m}}{\partial x_{j}}(X) u_{m}\right\|_{H^{1 / 4}\left(0, T ; L^{2}(\partial \Omega)\right)} \leqslant C T^{1 / 8}\left\|\frac{\partial a_{k m}}{\partial x_{j}}(X)\right\|_{H^{7 / 8}\left(0, T ; L^{8 / 3}(\partial \Omega)\right)}\left\|u_{m}\right\|_{H^{1 / 4}\left(0, T ; L^{8}(\partial \Omega)\right)} \leqslant C_{R} T^{1 / 8},
$$

where we have used A.5 and

$$
\frac{\partial a_{k m}}{\partial x_{j}}(X)=0 \quad \text { at } t=0
$$

All the other terms are estimated similarly so that we finally deduce $(6.32)$. The estimate $(6.31)$ on $H$ can be done similarly as the estimate 6.32 for $G$.

\subsection{Proof of Theorem 6.1}

We are now in position to prove Theorem 6.1.

Proof of Theorem 6.1. First let us prove the local in time existence. We recall that $\Phi$ is given by $(6.5)$, with $\mathcal{Y}_{T}$ given by 6.1). From 6.30, 6.32, (6.31), we obtain

$$
\|\Phi(f, \widetilde{g}, h)\|_{\mathcal{Y}_{T}} \leqslant C_{R} T^{1 / 8} .
$$

Thus, for $T$ small enough, we obtain that $\Phi\left(\mathcal{B}_{T, R}\right) \subset \mathcal{B}_{T, R}$, where $\mathcal{B}_{T, R}$ is defined by 6.2. With computations similar as the ones done in the two previous subsections, we also obtain that for $T$ small enough, $\left.\Phi\right|_{\mathcal{B}_{T, R}}$ is a contraction. Using the Banach fixed-point theorem, we deduce the existence and uniqueness of $(u, p, \eta)$ solution of the system (3.5), (3.6) and (3.7) provided that $T$ is small enough.

For the second part of Theorem 6.1, the application $\Phi$ is defined in a similar way as 6.5 but with $T=\infty$ and

$$
\mathcal{Y}_{\infty}=L_{\gamma}^{2}\left(0, \infty ;\left[L^{2}(\Omega)\right]^{3}\right) \times W_{\gamma}^{1 / 4}\left(0, \infty ;\left[H^{1 / 2}(\partial \Omega)\right]^{3},\left[L^{2}(\partial \Omega)\right]^{3}\right) \times L_{\gamma}^{2}\left(0, \infty ; L^{2}(\omega)\right)
$$

Here $\gamma \in\left[0, \gamma_{0}\right]$, where $\gamma_{0}$ is given by Theorem 5.4. In that case, we show that for $R$ small enough $\Phi\left(\mathcal{B}_{\infty, R}\right) \subset$ $\mathcal{B}_{\infty, R}$ and that $\Phi_{\mid \mathcal{B}_{\infty, R}}$ is a strict contraction. The estimates are similar to the previous case, but are simpler: for instance, Lemma 6.2 is replaced by the following estimates:

$$
\|\eta\|_{L_{\gamma}^{\infty}\left(0, \infty ; L^{\infty}(\omega)\right)}+\left\|\partial_{s_{j}} \eta\right\|_{L_{\gamma}^{\infty}\left(0, \infty ; L^{\infty}(\omega)\right)}+\left\|\partial_{s_{j} s_{k}}^{2} \eta\right\|_{L_{\gamma}^{\infty}\left(0, \infty ; L^{4}(\omega)\right)} \leqslant C\|\eta\|_{L_{\gamma}^{\infty}\left(0, \infty ; H^{3}(\omega)\right)} \leqslant C R
$$

In particular, there exists $R_{0}>0$ so that, if $R \leqslant R_{0}$, then

$$
\left\|\frac{1}{1+\eta}\right\|_{L^{\infty}\left(0, T ; L^{\infty}(\omega)\right)} \leqslant C .
$$

We can then define the changes of variables $X$ and $Y$ by 3.3 , and obtain similar estimates as in Lemma 6.3 . Lemma 6.4 and Proposition 6.5.

This yields

$$
\|\Phi(f, \widetilde{g}, h)\|_{\mathcal{Y}_{\infty}} \leqslant C R^{2},
$$


and

$$
\left\|\Phi\left(f^{(1)}, \widetilde{g}^{(1)}, h^{(1)}\right)-\Phi\left(f^{(2)}, \widetilde{g}^{(2)}, h^{(2)}\right)\right\|_{\mathcal{Y}_{\infty}} \leqslant C R\left\|\left(f^{(1)}, \widetilde{g}^{(1)}, h^{(1)}\right)-\left(f^{(2)}, \widetilde{g}^{(2)}, h^{(2)}\right)\right\|_{\mathcal{Y}_{\infty}} .
$$

for $(f, \widetilde{g}, h),\left(f^{(i)}, \widetilde{g}^{(i)}, h^{(i)}\right) \in \mathcal{B}_{\infty, R}$. Then, we use the Banach fixed point by taking $R$ small enough and we deduce the global existence and uniqueness of a strong solution $(u, p, \eta) \in \mathcal{X}_{\infty, \gamma}$ for the system [3.5, , 3.6. and (3.7) provided that $R$ is small enough.

\section{A Technical results}

In this section, we give some technical estimates that have been elaborated in [6]. Given a function $\xi$, we define for $t^{*} \in[0,1], \xi^{*}\left(t^{*}\right)=\xi\left(t^{*} T\right)$. Assume $\mathfrak{X}$ is a Banach space. If $\xi \in H^{s}(0, T ; \mathfrak{X})$, then $\xi^{*} \in H^{s}(0,1 ; \mathfrak{X})$ and

$$
\left\lfloor\xi^{*}\right\rfloor_{s, 2,(0,1), \mathfrak{X}}=T^{(2 s-1) / 2}\lfloor\xi\rfloor_{s, 2,(0, T), \mathfrak{x}} .
$$

Assume $\sigma_{2} \in(1 / 2,1]$ and $\sigma_{1} \in\left[0, \sigma_{2}\right]$. Using the above result, there exists a constant independent of $T$ such that for any $\xi \in H^{\sigma_{2}}(0, T ; \mathfrak{X})$ and $\xi(0)=0$, then

$$
\|\xi\|_{H^{\sigma_{1}(0, T, \mathfrak{x})}} \leqslant C T^{\sigma_{2}-\sigma_{1}}\|\xi\|_{H^{\sigma_{2}(0, T, \mathfrak{X})}} .
$$

We also recall the following result on the interpolation estimates (with constants independent of $T$ ), see [6. Lemma A.5]: assume $\sigma \in[0,1], \mu_{1} \geqslant 0, \mu_{2} \geqslant 0$ and $\mu=\sigma \mu_{1}+(1-\sigma) \mu_{2}$. Then there exists a constant $C$ independent of $T$ such that for any function $u \in H^{1}\left(0, T ; H^{\mu_{1}}(\Omega)\right) \cap L^{2}\left(0, T ; H^{\mu_{2}}(\Omega)\right)$, we have

$$
\|u\|_{H^{\sigma}\left(0, T ; H^{\mu}(\Omega)\right)} \leqslant C\|u\|_{H^{1}\left(0, T ; H^{\mu_{1}}(\Omega)\right)}^{\sigma}\|u\|_{L^{2}\left(0, T ; H^{\mu_{2}}(\Omega)\right)}^{1-\sigma} .
$$

On the other hand, for $p, q \in[1,+\infty]$ and $\frac{1}{r}=\frac{\sigma}{p}+\frac{(1-\sigma)}{q}$, we have

$$
\|u\|_{L^{r}\left(0, T ; H^{\mu}(\Omega)\right)} \leqslant C\|u\|_{L^{p}\left(0, T ; H^{\left.\mu_{1}(\Omega)\right)}\right.}^{\sigma}\|u\|_{L^{q}\left(0, T ; H^{\left.\mu_{2}(\Omega)\right)}\right.}^{1-\sigma},
$$

for $u \in L^{p}\left(0, T ; H^{\mu_{1}}(\Omega)\right) \cap L^{q}\left(0, T ; H^{\mu_{2}}(\Omega)\right)$.

We give also a useful formula (see [6, Lemma A.7]) for the product of functions: assume that $\mathfrak{X}_{1}, \mathfrak{X}_{2}$ and $\mathfrak{X}_{3}$ are Banach spaces such that

$$
\|f g\|_{\mathfrak{X}_{3}} \leqslant C\|f\|_{\mathfrak{X}_{1}}\|g\|_{\mathfrak{X}_{2}}, \quad \forall f \in \mathfrak{X}_{1}, \quad \forall g \in \mathfrak{X}_{2} .
$$

Let us assume $\sigma \in(1 / 2,1], s \in[0,1 / 2], T_{0}>0$. Then there exists a constant $C$ such that for any $T \leqslant T_{0}$ we have

$$
\left\|u_{1} u_{2}\right\|_{H^{s}\left(0, T ; \mathfrak{x}_{3}\right)} \leqslant C T^{\sigma-s-1 / 2}\left\|u_{1}\right\|_{H^{s}\left(0, T ; \mathfrak{x}_{1}\right)}\left\|u_{2}\right\|_{H^{\sigma}\left(0, T ; \mathfrak{X}_{2}\right)}+\left\|u_{2}(0)\right\|_{\mathfrak{X}_{2}}\left\|u_{1}\right\|_{H^{s}\left(0, T ; \mathfrak{x}_{1}\right)},
$$

for all $u_{1} \in H^{s}\left(0, T ; \mathfrak{X}_{1}\right)$ and $u_{2} \in H^{\sigma}\left(0, T ; \mathfrak{X}_{2}\right)$.

\section{References}

[1] Paul Acevedo, Cherif Amrouche, Carlos Conca, and Amrita Ghosh. Stokes and navier-stokes equations with navier boundary condition. https://arxiv.org/abs/1805.07760v1, 2018.

[2] Mehdi Badra and Takéo Takahashi. Feedback boundary stabilization of 2D fluid-structure interaction systems. Discrete Contin. Dyn. Syst., 37(5):2315-2373, 2017.

[3] H. Beirão da Veiga. On the existence of strong solutions to a coupled fluid-structure evolution problem. J. Math. Fluid Mech., 6(1):21-52, 2004. 
[4] H. Beirão Da Veiga. Regularity for Stokes and generalized Stokes systems under nonhomogeneous slip-type boundary conditions. Adv. Differential Equations, 9(9-10):1079-1114, 2004.

[5] Alain Bensoussan, Giuseppe Da Prato, Michel C. Delfour, and Sanjoy K. Mitter. Representation and control of infinite dimensional systems. Systems \& Control: Foundations \& Applications. Birkhäuser Boston, Inc., Boston, MA, second edition, 2007.

[6] Muriel Boulakia, Sergio Guerrero, and Takeo Takahashi. Well-posedness for the coupling between a viscous incompressible fluid and an elastic structure. https://hal.inria.fr/hal-01939464, November 2018. Preprint.

[7] Marco Bravin. On the weak uniqueness of "viscous incompressible fluid + rigid body" system with Navier slip-with-friction conditions in a 2D bounded domain. https://hal.archives-ouvertes.fr/ hal-01740859, March 2018. Preprint.

[8] Loredana Bălilescu, Jorge San Martín, and Takéo Takahashi. Fluid-rigid structure interaction system with Coulomb's law. SIAM J. Math. Anal., 49(6):4625-4657, 2017.

[9] Jean-Jérôme Casanova. Existence of Time-Periodic Strong Solutions to a Fluid-Structure System. https: //hal.archives-ouvertes.fr/hal-01838262, July 2018. Preprint.

[10] Jean-Jérôme Casanova. Fluid structure system with boundary conditions involving the pressure. https: //arxiv.org/abs/1707.06382, 2017.

[11] Shu Ping Chen and Roberto Triggiani. Proof of extensions of two conjectures on structural damping for elastic systems. Pacific J. Math., 136(1):15-55, 1989.

[12] David Gérard-Varet and Matthieu Hillairet. Existence of weak solutions up to collision for viscous fluid-solid systems with slip. Comm. Pure Appl. Math., 67(12):2022-2075, 2014.

[13] David Gérard-Varet, Matthieu Hillairet, and Chao Wang. The influence of boundary conditions on the contact problem in a 3D Navier-Stokes flow. J. Math. Pures Appl. (9), 103(1):1-38, 2015.

[14] Céline Grandmont. On an unsteady fluid-beam interaction problem. https://basepub.dauphine.fr/ bitstream/handle/123456789/6848/2004-48.pdf 2004. Preprint.

[15] Céline Grandmont and Matthieu Hillairet. Existence of global strong solutions to a beam-fluid interaction system. Arch. Ration. Mech. Anal., 220(3):1283-1333, 2016.

[16] Giovanna Guidoboni, Marcello Guidorzi, and Mariarosaria Padula. Continuous dependence on initial data in fluid-structure motions. J. Math. Fluid Mech., 14(1):1-32, 2012.

[17] M. Guidorzi, M. Padula, and P. I. Plotnikov. Hopf solutions to a fluid-elastic interaction model. Math. Models Methods Appl. Sci., 18(2):215-269, 2008.

[18] M. Hillairet. Lack of collision between solid bodies in a 2D incompressible viscous flow. Comm. Partial Differential Equations, 32(7-9):1345-1371, 2007.

[19] Matthieu Hillairet and Takéo Takahashi. Collisions in three-dimensional fluid structure interaction problems. SIAM J. Math. Anal., 40(6):2451-2477, 2009.

[20] C. O. Horgan. Korn's inequalities and their applications in continuum mechanics. SIAM Rev., 37(4):491511, 1995.

[21] Atsushi Inoue and Minoru Wakimoto. On existence of solutions of the Navier-Stokes equation in a time dependent domain. J. Fac. Sci. Univ. Tokyo Sect. IA Math., 24(2):303-319, 1977. 
[22] S. F. Kistler and L. E. Scriven. Coating flow theory by finite element and asymptotic analysis of the Navier-Stokes system. International Journal for Numerical Methods in Fluids, 4(3):207-229, 1984.

[23] Julien Lequeurre. Existence of strong solutions to a fluid-structure system. SIAM J. Math. Anal., 43(1):389410, 2011.

[24] A. Liakos. Finite-element approximation of viscoelastic fluid flow with slip boundary condition. Comput. Math. Appl., 49(2-3):281-294, 2005.

[25] J.-L. Lions and E. Magenes. Problèmes aux limites non homogènes et applications. Vol. 2. Travaux et Recherches Mathématiques, No. 18. Dunod, Paris, 1968.

[26] Zhuangyi Liu and Songmu Zheng. Semigroups associated with dissipative systems, volume 398 of Chapman $\&$ Hall/CRC Research Notes in Mathematics. Chapman \& Hall/CRC, Boca Raton, FL, 1999.

[27] Boris Muha and Sunčica Čanić. Existence of a weak solution to a fluid-elastic structure interaction problem with the Navier slip boundary condition. J. Differential Equations, 260(12):8550-8589, 2016.

[28] CLMH Navier. Mémoire sur les lois du mouvement des fluides. Mémoires de l'Académie Royale des Sciences de l'Institut de France, 6(1823):389-440, 1823.

[29] Gabriela Planas and Franck Sueur. On the "viscous incompressible fluid + rigid body" system with Navier conditions. Ann. Inst. H. Poincaré Anal. Non Linéaire, 31(1):55-80, 2014.

[30] Jean-Pierre Raymond. Feedback stabilization of a fluid-structure model. SIAM J. Control Optim., 48(8):5398-5443, 2010.

[31] Jorge Alonso San Martín, Victor Starovoitov, and Marius Tucsnak. Global weak solutions for the twodimensional motion of several rigid bodies in an incompressible viscous fluid. Arch. Ration. Mech. Anal., 161(2):113-147, 2002.

[32] Rieko Shimada. On the $L_{p}-L_{q}$ maximal regularity for Stokes equations with Robin boundary condition in a bounded domain. Math. Methods Appl. Sci., 30(3):257-289, 2007.

[33] Roger Temam. Navier-Stokes equations, volume 2 of Studies in Mathematics and its Applications. NorthHolland Publishing Co., Amsterdam-New York, revised edition, 1979. Theory and numerical analysis, With an appendix by F. Thomasset.

[34] Roger Temam. Problèmes mathématiques en plasticité, volume 12 of Méthodes Mathématiques de l'Informatique [Mathematical Methods of Information Science]. Gauthier-Villars, Montrouge, 1983.

[35] Rüdiger Verfürth. Finite element approximation of incompressible Navier-Stokes equations with slip boundary condition. Numer. Math., 50(6):697-721, 1987.

[36] Chao Wang. Strong solutions for the fluid-solid systems in a 2-D domain. Asymptot. Anal., 89(3-4):263-306, 2014. 